Pacific

Journal of

Mathematics

\title{
PRIMITIVELY GENERATED HALL ALGEBRAS
}

ARKady Berenstein AND Jacob GREENSTEIN

Volume $281 \quad$ No. 2

April 2016 


\title{
PRIMITIVELY GENERATED HALL ALGEBRAS
}

\author{
ARKADY BERENSTEIN AND JACOB GREENSTEIN \\ Dedicated to Professor Anthony Joseph on the occasion of his seventieth birthday
}

\begin{abstract}
In the present paper we show that Hall algebras of finitary exact categories behave like quantum groups in the sense that they are generated by indecomposable objects. Moreover, for a large class of such categories, Hall algebras are generated by their primitive elements, with respect to the natural comultiplication, even for nonhereditary categories. Finally, we introduce certain primitively generated subalgebras of Hall algebras and conjecture an analogue of "Lie correspondence" for those finitary categories.
\end{abstract}

1. Introduction 287

2. Definitions and main results 292

3. Examples 298

4. The PBW property of Hall algebras and proof of Theorem 2.4309

5. The Grothendieck monoid of a profinitary category 315

6. Coalgebras in tensor categories and proof of the main theorem 318

7. Proof of Theorem $2.26 \quad 325$

Acknowledgments 328

References $\quad 328$

\section{Introduction}

It is well-known that quantum groups are not groups, but rather Hopf algebras, which are similar to enveloping algebras of Lie algebras. Hall-Ringel algebras $H_{\mathscr{A}}$ of finitary exact categories can be regarded, from many points of view, as generalizations of quantum groups. One aspect of this analogy is the following striking result, which we failed to find in the literature.

Theorem 1.1. The Hall algebra $H_{\mathscr{A}}$ of any finitary exact category $\mathscr{A}$ is generated by isomorphism classes of indecomposable objects in $\mathscr{A}$.

The authors were partially supported by NSF grants DMS-0800247, DMS-1101507 (Berenstein) and DMS-0654421 (Greenstein) and Simons foundation collaboration grant 245735 (Greenstein).

MSC2010: 16G20, 17B37, 16T15, 16T20.

Keywords: Hall algebra, exact category, PBW property, Nichols algebra. 
We prove a refinement of this theorem (Theorem 2.4), which is an analogue of the Poincaré-Birkhoff-Witt property for $H_{\mathscr{A}}$, in $\S 4.3$.

However, isomorphism classes of indecomposable objects are not the most efficient as a generating set. For example, if $\mathscr{A}$ is the representation category of a (valued) Dynkin quiver $Q$, then indecomposables correspond to all positive roots of the simple Lie algebra associated with $Q$, while $H_{\mathscr{A}}$ can be generated by simple objects (in other words, indecomposables corresponding to simple roots of the Lie algebra). Having this in mind, we introduce minimal generating sets for $H_{\mathscr{A}}$, namely, primitive elements, which generalize these simple root generators.

More precisely, for any finitary exact category $\mathscr{A}$, the Hall algebra $H_{\mathscr{A}}$ has a natural coproduct $\Delta: H_{\mathscr{A}} \rightarrow H_{\mathscr{A}} \widehat{\otimes} H_{\mathscr{A}}$ whose image may lie in a suitable completion of the tensor square of $H_{\mathscr{A}}$. Note, however, that the multiplication and $\Delta$ are not always compatible, that is, $\Delta$ need not be a homomorphism of algebras. The compatibility is guaranteed by Green's theorem (see [Green 1995]) for all hereditary cofinitary (so that $\Delta$ is an "honest" comultiplication rather than a topological one) abelian categories $\mathscr{A}$ (see Definition 2.11). This includes all categories $\operatorname{rep}_{\mathbb{k}} Q$ of finite dimensional representations over a finite field $\mathbb{k}$ of an acyclic (valued) quiver $Q$. In a remarkable paper, Sevenhant and Van den Bergh [2001] proved that for $\mathscr{A}=\operatorname{rep}_{\mathbb{k}} Q$ the Hall algebra $H_{\mathscr{A}}$ is a Nichols algebra in an appropriate braided tensor category (see $\$ 2.6$ for details) and, in particular, is generated by its space of primitive elements

$$
V_{\mathscr{A}}=\left\{v \in H_{\mathscr{A}}: \Delta(v)=v \otimes 1+1 \otimes v\right\} .
$$

We extend this result to a much larger class of categories that we refer to as profinitary categories. We introduce profinitary categories in terms of their Grothendieck monoids (denoted $\Gamma_{\mathscr{A}}$ for an exact category $\mathscr{A}$, see $\S 2.3$ for precise definitions) by requiring that groups of morphisms between any two objects and all Grothendieck equivalence classes are finite. By definition, $H_{\mathscr{A}}$ is naturally graded by $\Gamma_{\mathscr{A}}$ and if $\mathscr{A}$ is profinitary, all homogeneous components $\left(H_{\mathscr{A}}\right)_{\gamma}, \gamma \in \Gamma_{\mathscr{A}}$ are finite dimensional.

The class of profinitary categories is large enough. For instance, it includes the abelian category $R$ - fin of all finite $R$-modules $M$ (i.e., finite abelian groups with $R$-action) for a finitary unital ring $R$, as defined in [Ringel 1990a, §1]. This includes all finitely generated (over $\mathbb{Z}$ ) unital rings. Moreover, if $\mathscr{A}$ is profinitary, then so is any full subcategory $\mathscr{B} \subset \mathscr{A}$ closed under extensions. The following is the main result of the present work.

Main Theorem 1.2. For any profinitary and cofinitary exact category $\mathscr{A}$, the Hall algebra $H_{\mathscr{A}}$ is generated by the space $V_{\mathscr{A}}$ of its primitive elements. Moreover, $V_{\mathscr{A}}$ is minimal in the sense that a nonzero element of $V_{\mathscr{A}}$ cannot be expressed as a sum of products of elements of $V_{\mathscr{A}}$. 
We prove Main Theorem 1.2 in $§ 6.4$.

Based on the second assertion of Main Theorem 1.2, we can introduce quasiNichols algebras as both algebras and coalgebras minimally generated by their primitive elements (see Definition 2.17 for details). In particular, it is easy to see (cf. Lemma 2.25) that any Nichols algebra is quasi-Nichols. It is noteworthy that the minimality of $V_{\mathscr{A}}$ has the following nice consequence for constructing primitive elements in $H_{\mathscr{A}}$ : once we find a subspace $U$ of $V_{\mathscr{A}}$ such that $U$ generates $H_{\mathscr{A}}$ as an algebra, we must stop because $U$ is the space of all primitive elements in $H_{\mathscr{A}}$.

Remark 1.3. Similarly to Grothendieck groups, exact functors induce canonical homomorphisms of Grothendieck monoids. However, even for full embeddings, such homomorphisms need not be injective. On the other hand, unlike the Grothendieck group, the Grothendieck monoid always separates simple objects of the category. For instance, if $\mathscr{A}$ is the category of $\mathbb{k}$-representations of the quiver $Q=1 \rightarrow 2$ with dimension vectors $(n, 2 n), n \in \mathbb{Z}_{\geq 0}$, then $K_{0}(\mathscr{A}) \cong \mathbb{Z}$, but $\Gamma_{\mathscr{A}}$ is an additive monoid generated by $\beta_{1}, \beta_{2}$ subject to the relations $\beta_{1}+\beta_{2}=2 \beta_{1}=2 \beta_{2}$. The canonical homomorphism $\Gamma_{\mathscr{A}} \rightarrow K_{0}(\mathscr{A})$ is given by $\beta_{1} \mapsto 1, \beta_{2} \mapsto 1$ and thus is not injective (see $\S 3.4$ for details.) It should also be noted that in this example $\Gamma_{\mathscr{A}}$ is not a submonoid of the Grothendieck monoid of the category $\operatorname{rep}_{\mathbb{k}} Q$ since in $\Gamma_{\text {rep }_{k} Q}$ both simple objects of $\mathscr{A}$ belong to the same class.

A nice property of profinitary categories is that their Hall algebras always contain primitive elements. If $\mathscr{A}$ is profinitary, then its Grothendieck monoid admits a natural partial order and is generated by its minimal elements with respect to that order (Proposition 2.12). Moreover, for $\gamma$ minimal the corresponding homogeneous component $\left(H_{\mathscr{A}}\right)_{\gamma}$ of $H_{\mathscr{A}}$ is one-dimensional and primitive.

Quite surprisingly, for a profinitary category, cofinitarity is a simple property of its Grothendieck monoid. We say that a monoid $\Gamma$ is locally finite if for all $\gamma \in \Gamma$, the set $\{(\alpha, \beta) \in \Gamma \times \Gamma: \alpha+\beta=\gamma\}$ is finite.

Theorem 1.4. A profinitary exact category $\mathscr{A}$ is cofinitary if and only if $\Gamma_{\mathscr{A}}$ is locally finite.

We prove this theorem in $\S 5.3$. As a corollary, we obtain two classes of categories for which profinitarity implies cofinitarity.

Corollary 1.5. (a) Any full exact subcategory of a profinitary abelian category is cofinitary.

(b) Any profinitary exact category whose Grothendieck monoid is finitely generated is cofinitary.

This corollary is proven in $§ 5.3$. Based on the above, we propose the following conjecture. 
Conjecture 1.6. For any profinitary exact category $\mathscr{A}$, its Grothendieck monoid $\Gamma_{\mathscr{A}}$ is locally finite.

By Theorem 1.4, any category as in the above conjecture is also cofinitary.

This conjecture is nontrivial since there exist profinitary exact categories $\mathscr{A}$ for which any ambient abelian category $\overline{\mathscr{A}}$ (which always exists, see, e.g., [Bühler 2010; Keller 1990]) is not profinitary, and the monoid $\Gamma_{\mathscr{A}}$ need not be finitely generated.

Main Theorem 1.2 and Corollary 1.5(a) imply the following theorem.

Theorem 1.7. If $\mathscr{A}$ is a profinitary hereditary abelian category, then $H_{\mathscr{A}}$ is a Nichols algebra (see Definition 2.23) of the (braided) space $V_{\mathscr{A}}$ of its primitive elements.

We prove a refined version of this statement (Theorem 2.26) in §7.2.

The case when $\mathscr{A}=\operatorname{rep}_{\mathbb{k}} Q$ where $Q$ is a finite acyclic (valued) quiver was established in [Sevenhant and Van Den Bergh 2001, Theorem 1.1], which inspired the present work. If $\mathscr{A}$ is the category of nilpotent representations of $\mathbb{k}[x]$ for a finite field $\mathbb{k}$, then Theorem 1.7 recovers the classical result of Zelevinsky [1981] that the Hall-Steinitz algebra is a Hopf algebra (see, e.g., $\$ 3.1$ for details). More generally, it is well-known that the category $\operatorname{rep}_{\mathbb{k}} Q$ for any finite valued quiver $Q$ is hereditary (see [Gabriel 1973; Hubery 2007]). Therefore, Theorem 1.7 is applicable to such a category as well, that is, $H_{\text {rep }_{k} Q}$ is a Nichols algebra. In particular, so is the Hall algebra of the category of finite dimensional modules of the free algebra in $n$ generators over $\mathbb{k}$.

Furthermore, by definition, $V_{\mathscr{A}}$ is graded by $\Gamma_{\mathscr{A}}$, that is, $V_{\mathscr{A}}=\bigoplus_{\gamma \in \Gamma}\left(V_{\mathscr{A}}\right)_{\gamma}$, so $\gamma \in \Gamma_{\mathscr{A}}$ with $\left(V_{\mathscr{A}}\right)_{\gamma} \neq 0$ can be thought of as "simple roots" of $\mathscr{A}$. Given $\gamma \in \Gamma_{\mathscr{A}}^{+}$, define its multiplicity $m_{\gamma}$ by

$$
m_{\gamma}:=\# \text { Ind } \mathscr{A}_{\gamma}-\operatorname{dim}_{\mathbb{Q}}\left(V_{\mathscr{A}}\right)_{\gamma},
$$

where Ind $\mathscr{A}_{\gamma}=$ Ind $\mathscr{A} \cap$ Iso $\mathscr{A}_{\gamma}$. This definition is justified by the following proposition.

Proposition 1.8. Let $\mathscr{A}$ be a profinitary cofinitary exact category. Then $m_{\gamma} \geq 0$ for all $\gamma \in \Gamma_{\mathscr{A}}^{+}$.

We prove a more precise version of this result (Proposition 2.20) in §6.5. In particular, Proposition 1.8 implies that if Ind $\mathscr{A}_{\gamma}=\varnothing$ then $\left(V_{\mathscr{A}}\right)_{\gamma}=0$, that is, we should look for primitive elements only in those graded components where indecomposables live. Moreover, if Ind $\mathscr{A}$ is finite, then obviously $V_{\mathscr{A}}$ is finite dimensional and we have an efficient procedure for computing it (see §3).

The term "multiplicity" is justified by the following result, which is an immediate consequence of reformulations [Hua 2000, Theorem 4.1; Deng and Xiao 2003, $\S 4.1]$ of the famous Kac conjecture [Kac 1980], proved in [Hausel 2010]. 
Theorem 1.9. Let $Q$ be an acyclic quiver, $\mathfrak{g}_{Q}$ be the corresponding Kac-Moody algebra and $\mathscr{A}=\operatorname{rep}_{\mathbb{k}}(Q)$ where $\mathbb{k}$ is a finite field with $q$ elements. Then for any $\gamma \in \Gamma_{\mathscr{A}}$ one has:

(a) $m_{\gamma}>0$ if and only if $\gamma$ is a nonsimple positive root of $\mathfrak{g}_{Q}$; in that case, $m_{\gamma}=\operatorname{dim}\left(\mathfrak{g}_{Q}\right)_{\gamma}$, that is $m_{\gamma}$ is the multiplicity of the root $\gamma$ in $\mathfrak{g}_{Q}$.

(b) $\left(V_{\mathscr{A}}\right)_{\gamma}=0$ unless $\gamma$ is simple or imaginary.

(c) For any imaginary root $\gamma$ of $\mathfrak{g}_{Q}, \operatorname{dim}_{\mathbb{Q}}\left(V_{\mathscr{A}}\right)_{\gamma}=p_{\gamma}(q)$ where $p_{\gamma} \in x \mathbb{Q}[x]$.

In view of Theorem 1.9(c) and results of [Sevenhant and Van Den Bergh 2001] we define real simple roots of $\mathscr{A}$ to be elements $\gamma \in \Gamma_{\mathscr{A}}$ for which $\operatorname{dim}_{\mathbb{Q}}\left(V_{\mathscr{A}}\right)_{\gamma}=1$ and imaginary simple roots of $\mathscr{A}$ to be those $\gamma \in \Gamma_{\mathscr{A}}$ with $\operatorname{dim}_{\mathbb{Q}}\left(V_{\mathscr{A}}\right)_{\gamma} \geq 2$. For a profinitary category $\mathscr{A}$ we show (Lemma 5.3) that all minimal elements of $\Gamma_{\mathscr{A}} \backslash\{0\}$ are real simple roots.

In fact, the consideration of examples suggests that a stronger version of this statement holds.

Conjecture 1.10. Let $\mathscr{A}$ be a profinitary and cofinitary exact category. Then each simple imaginary root of $\mathscr{A}$ has nonzero multiplicity.

Clearly, Theorem 1.9 verifies this conjecture when $\mathscr{A}=\operatorname{rep}_{\mathbb{k}}(Q)$ for any finite acyclic quiver $Q$. We provide more supporting evidence in $\S 3$. In those cases, $m_{\gamma}=1$ quite frequently (see $\S 3.2, \S 3.3$ and $\S 3.4$ ).

Simple real roots are of special interest. Denote by $U_{\mathscr{A}}$ the subalgebra of $H_{\mathscr{A}}$ generated by all $\left(V_{\mathscr{A}}\right)_{\alpha}$, where $\alpha$ runs over all real simple roots of $\mathscr{A}$, and refer to it as the quantum enveloping algebra of $\mathscr{A}$. The following well-known fact justifies this definition.

Theorem 1.11 [Ringel 1990b]. If $Q$ is an acyclic valued quiver, then $U_{\operatorname{rep}_{\mathbb{k}} Q}$ is isomorphic to a quantized enveloping algebra of the nilpotent part of $\mathfrak{g}_{Q}$.

Since $[X] \in$ Iso $\mathscr{A}$ is primitive if and only if it is almost simple (see Definition 5.2), the algebra $U_{\mathscr{A}}$ contains the subalgebra $C_{\mathscr{A}}$ of $H_{\mathscr{A}}$ generated by isomorphism classes of all almost simple objects. We call $C_{\mathscr{A}}$ the composition algebra of $\mathscr{A}$ since it generalizes the composition algebra of $\operatorname{rep}_{\mathbb{k}} Q$, which is the subalgebra of $H_{\text {rep }_{k}} Q$ generated by isomorphism classes of simple objects. In fact, in the assumptions of the above theorem, $U_{\mathrm{rep}_{k} Q}=C_{\mathrm{rep}_{\mathrm{k}} Q}$. However, it frequently happens that $C_{\mathscr{A}} \subsetneq U_{\mathscr{A}}$ (see $\S 3$ for examples). Note the following corollary of Theorem 1.7 and [Andruskiewitsch and Schneider 2002, Corollary 2.3] (see Lemma 2.24).

Corollary 1.12. If $\mathscr{A}$ is a profinitary hereditary abelian category then both $C_{\mathscr{A}}$ and $U_{\mathscr{A}}$ are Nichols algebras. 
It turns out that there is another algebra $E_{\mathscr{A}}$, which (yet conjecturally) "squeezes" between these two. That is, $E_{\mathscr{A}}$ is generated by elements $e_{\gamma} \in H_{\mathscr{A}}$, where $e_{\gamma}$ is the sum of all isomorphism classes of objects of $\mathscr{A}$ whose image in $\Gamma$ is $\gamma$. Since

$$
\operatorname{Exp}_{\mathscr{A}}:=\sum_{\gamma \in \Gamma_{A}} e_{\gamma}
$$

is a group-like element in the completion of $H_{\mathscr{A}}$ with respect to a slightly different coproduct (see [Berenstein and Greenstein 2013, Lemma A.1]), we referred to $\operatorname{Exp}_{\mathscr{A}}$ in [Berenstein and Greenstein 2013] as the exponential of $\mathscr{A}$. Hence we sometimes refer to $E_{\mathscr{A}}$ as the exponential algebra of $\mathscr{A}$. By definition, $C_{\mathscr{A}} \subset E_{\mathscr{A}}$.

Conjecture 1.13. For any profinitary category $\mathscr{A}$ one has

$$
E_{\mathscr{A}}=U_{\mathscr{A}} \text {. }
$$

In particular, $\operatorname{Exp}_{\mathscr{A}}$ belongs to the completion of $U_{\mathscr{A}}$.

In $\S 3$ we provide several supporting examples of profinitary categories $\mathscr{A}$ together with the explicit presentations of $H_{\mathscr{A}}, U_{\mathscr{A}}$ and $E_{\mathscr{A}}$.

The significance of the conjecture is that it paves the ground for the "Lie correspondence" between the enveloping algebra $U_{\mathscr{A}}$ and the quantum Chevalley group $G_{\mathscr{A}}$ that we introduced in [Berenstein and Greenstein 2013] as an analogue of the corresponding Lie group. That is, Conjecture 1.13 implies that the "tame" part of $G_{\mathscr{A}}$ belongs to the completion of $U_{\mathscr{A}}$.

\section{Definitions and main results}

2.1. Exact categories and Hall algebras. All categories are assumed to be essentially small. For such a category $\mathscr{A}$ we denote by Iso $\mathscr{A}$ the set of isomorphism classes of objects in $\mathscr{A}$. We say that a category $\mathscr{A}$ is Hom-finite if $\operatorname{Hom}_{\mathscr{A}}(X, Y)$ is a finite set for all $X, Y \in \mathscr{A}$.

Let $\mathscr{A}$ be an exact category, in the sense of [Quillen 1973] (see also [Keller 1990; Bühler 2010]). We denote by $\operatorname{Ext}_{\mathscr{A}}^{1}(A, B)$ the set of all isomorphism classes $[X] \in$ Iso $\mathscr{A}$ such that there exists a short exact sequence

$$
B \stackrel{f}{\longrightarrow} X \stackrel{g}{\longrightarrow} A
$$

(here $f$ is a monomorphism, $g$ is an epimorphism, $f$ is a kernel of $g$ and $g$ is a cokernel of $f$ ). We say that $\mathscr{A}$ is finitary if it is $\operatorname{Hom}$-finite and $\operatorname{Ext}_{\mathscr{A}}^{1}(A, B)$ is finite for every $A, B \in \mathscr{A}$.

Following [Hubery 2006] we define Hall numbers for finitary exact categories as follows. For $A, B, X \in \mathscr{A}$ fixed, denote by $\mathcal{E}(A, B)_{X}$ the set of all short exact sequences (2-1). The group $\operatorname{Aut}_{\mathscr{A}} A \times \operatorname{Aut}_{\mathscr{A}} B$ acts freely on $\mathcal{E}(A, B)_{X}$ by

$$
(\varphi, \psi) .(f, g)=\left(f \varphi^{-1}, \psi g\right), \quad \varphi \in \operatorname{Aut}_{\mathscr{A}} B, \psi \in \operatorname{Aut}_{\mathscr{A}} A .
$$


The Hall number $F_{A B}^{X}$ is the number of $\operatorname{Aut}_{\mathscr{A}} A \times \operatorname{Aut}_{\mathscr{A}} B$-orbits in $\mathcal{E}(A, B)_{X}$ and equals

$$
F_{A B}^{X}=\frac{\# \mathcal{E}(A, B)_{X}}{\#\left(\operatorname{Aut}_{\mathscr{A}} A \times \operatorname{Aut}_{\mathscr{A}} B\right)} .
$$

Denote

$$
H_{\mathscr{A}}=\mathbb{Q} \text { Iso } \mathscr{A}=\bigoplus_{[X] \in \operatorname{Iso} \mathscr{A}} \mathbb{Q} \cdot[X] .
$$

Proposition 2.1 [Ringel 1990a; Hubery 2006]. For any finitary exact category $\mathscr{A}$, the space $H_{\mathscr{A}}$ is an associative unital $\mathbb{Q}$-algebra with the product given by

$$
[A] \cdot[B]=\sum_{[C] \in \operatorname{Iso} \mathscr{A}} F_{A, B}^{C}[C] .
$$

The unity $1 \in H_{\mathscr{A}}$ is the class [0] of the zero object of $\mathscr{A}$.

It is well-known (see, e.g., [Bühler 2010; Keller 1990]) that each exact category $\mathscr{A}$ can be realized as a full subcategory closed under extensions of an abelian category $\overline{\mathscr{A}}$. However, even if $\mathscr{A}$ is finitary, it might be impossible to find an ambient abelian category which is also finitary. On the other hand, any full subcategory of a finitary abelian category closed under extensions is also finitary.

2.2. Ordered monoids and the PBW property of Hall algebras. Let $\Lambda$ be an abelian monoid. We say that $\Lambda$ is ordered if there exists a partial order $\triangleleft$ on $\Lambda^{+}$ such that for $\mu, \mu^{\prime}, v, v^{\prime} \in \Lambda^{+}$, we have

$$
\mu \triangleleft v, \mu^{\prime} \unlhd v^{\prime} \Longrightarrow \mu+\mu^{\prime} \triangleleft v+v^{\prime} .
$$

Let $\mathscr{A}$ be a finitary exact category. The set Iso $\mathscr{A}$ is naturally an abelian monoid with the addition operation defined by $[X]+[Y]=[X \oplus Y]$. Every object in $\mathscr{A}$ is a finite direct sum of indecomposable objects (see Lemma 4.9). Thus, in particular, Ind $\mathscr{A}$ generates Iso $\mathscr{A}$ as a monoid. The category $\mathscr{A}$ is said to be Krull-Schmidt if Iso $\mathscr{A}$ is freely generated by Ind $\mathscr{A}$.

Define a relation $\triangleleft$ on $(\text { Iso } \mathscr{A})^{+}$by $[M] \triangleleft[N]$ if

(i) $[N]=\left[M^{+} \oplus M^{-}\right]$, and

(ii) there exists a nonsplit short exact sequence $M^{-} \longmapsto M \rightarrow M^{+}$.

By abuse of notation, we also denote by $\triangleleft$ the transitive closure of this relation.

We say that a partial (pre)order $\prec$ on a set $\Lambda$ is inductive if there exists a function $f: \Lambda \rightarrow \mathbb{Z}_{\geq 0}$ such that $\lambda \prec \mu \Longrightarrow f(\lambda)<f(\mu)$. It is obvious that an inductive preorder is a partial order.

Theorem 2.2. Let $\mathscr{A}$ be a Hom-finite exact category. Then (Iso $\mathscr{A}, \triangleleft$ ) is an ordered monoid and $\triangleleft$ is inductive with the function $f:$ Iso $\mathscr{A} \rightarrow \mathbb{Z}_{\geq 0}$ given by

$$
f([M])=\# \operatorname{End}_{\mathscr{A}} M .
$$


Remark 2.3. If the category $\mathscr{A}$ is finitary, one can show that the assertion holds with $f$ replaced by the function $[M] \mapsto \# \operatorname{Ext}_{\mathscr{A}}^{1}(M, M),[M] \in$ Iso $\mathscr{A}$.

We prove this theorem in $\S 4.2$. It is used as the key ingredient in a proof of the following theorem, which generalizes [Guo and Peng 1997, Theorem 3.1] and establishes the (weak) PBW property of Hall algebras.

Theorem 2.4. Let $\mathscr{A}$ be a finitary exact category. Then for any total order on the set Ind $\mathscr{A}$ of isomorphism classes of indecomposable objects in $\mathscr{A}, H_{\mathscr{A}}$ is spanned, as a $\mathbb{Q}$-vector space, by ordered monomials on Ind $\mathscr{A}$. Moreover, if $\mathscr{A}$ is Krull-Schmidt, then such monomials form a basis of $H_{\mathscr{A}}$.

We prove this theorem in $\$ 4.3$. After [Joyce 2007; Riedtmann 1994], this further extends an analogy between Hall algebras of finitary categories and universal enveloping algebras.

2.3. The Grothendieck monoid and grading. Define the relation $\equiv$ on the monoid Iso $\mathscr{A}$ by

$$
[X] \equiv[Y] \Longleftrightarrow[X],[Y] \in \underline{\operatorname{Ext}}_{\mathscr{A}}^{1}(M, N) \text { for some } M, N \in \mathscr{A} .
$$

This relation is clearly symmetric and reflexive, hence its transitive closure is an equivalence relation on Iso $\mathscr{A}$ which we also denote by $\equiv$. The additivity of $\operatorname{Ext}_{\mathscr{A}}^{1}(A, B):=\bigcup_{X} \mathcal{E}(A, B)_{X} / \operatorname{Aut}_{\mathscr{A}} X$ in both $A$ and $B$ yields the following lemma.

Lemma 2.5. The relation $\equiv$ is a congruence relation on Iso $\mathscr{A}$, that is, $[X] \equiv[Y]$, $\left[X^{\prime}\right] \equiv\left[Y^{\prime}\right]$ implies that $\left[X \oplus X^{\prime}\right] \equiv\left[Y \oplus Y^{\prime}\right]$.

Definition 2.6. The Grothendieck monoid $\Gamma_{\mathscr{A}}$ of $\mathscr{A}$ is the quotient of Iso $\mathscr{A}$ by the congruence $\equiv$.

Given an object $M$ in $\mathscr{A}$, we denote its image in $\Gamma_{\mathscr{A}}$ by $|M|$. For all $\gamma \in \Gamma_{\mathscr{A}}$, set

$$
\text { Iso } \mathscr{A}_{\gamma}=\{[X] \in \text { Iso } \mathscr{A}:|X|=\gamma\} \text {. }
$$

We refer to Iso $\mathscr{A}_{\gamma}$ as a Grothendieck class in $\mathscr{A}$, and write Ind $\mathscr{A}_{\gamma}=$ Ind $\mathscr{A} \cap$ Iso $\mathscr{A}_{\gamma}$. The following fact is obvious.

Lemma 2.7. For any finitary exact category $\mathscr{A}$, the assignment $[M] \mapsto|M|$ defines a grading of the Hall algebra $H_{\mathscr{A}}$ of $\mathscr{A}$ by the Grothendieck monoid $\Gamma_{\mathscr{A}}$.

Remark 2.8. After Grothendieck, one defines the Grothendieck group $K_{0}(\mathscr{A})$ of $\mathscr{A}$ as the universal abelian group generated by $\Gamma_{\mathscr{A}}$. Note that the canonical homomorphism of monoids $\Gamma_{\mathscr{A}} \rightarrow K_{0}(\mathscr{A})$ can be very far from injective. One example was already provided in the introduction. Perhaps the most extreme example is the following. Let $\mathscr{A}=$ Vect $_{\mathfrak{k}}$ be the category of all $k$-vector spaces over some field $\mathbb{k}$. Then $\Gamma_{\mathscr{A}}$ identifies with the monoid of cardinal numbers. In particular, 
if $V$ is infinite dimensional and $W$ is finite dimensional then $|V|=|V|+|V|=$ $|W|+|V|$. This implies that in $K_{0}\left(\operatorname{Vect}_{\mathbb{k}}\right),|U|=0$ for every object $U$ of Vect $_{\mathbb{k}}$, that is, $K_{0}\left(\operatorname{Vect}_{\mathbb{k}}\right)=0$.

Also, while $K_{0}(\mathscr{A})$ can contain elements of finite order, this never occurs in $\Gamma_{\mathscr{A}}$. Indeed, since [0] $\in \underline{\operatorname{Ext}}_{\mathscr{A}}^{1}(A, B)$ implies that $A=B=0$ and the direct sum of two nonzero objects is clearly nonzero, we immediately obtain the following lemma.

Lemma 2.9. For any exact category $\mathscr{A}$, zero is the only invertible element of the Grothendieck monoid $\Gamma_{\mathscr{A}}$.

2.4. Profinitary and cofinitary categories. Let $\Gamma$ be an abelian monoid. Define a relation $\preceq$ on $\Gamma$ by $\alpha \preceq \beta$ if $\beta=\alpha+\gamma$ for some $\gamma \in \Gamma$. This relation is clearly an additive preorder and $0 \preceq \gamma$ for any $\gamma \in \Gamma$. The following lemma is obvious.

Lemma 2.10. The preorder $\preceq$ is a partial order on $\Gamma$ if and only if the equality $\alpha+\beta+\gamma=\alpha$ for $\alpha, \beta, \gamma \in \Gamma$ implies that $\alpha=\alpha+\beta=\alpha+\gamma$. In that case, 0 is the only invertible element of $\Gamma$.

We say that $\Gamma$ is naturally ordered if $\preceq$ is a partial order.

Definition 2.11. We say that a Hom-finite exact category $\mathscr{A}$ is

(i) profinitary if Iso $\mathscr{A}_{\gamma}$ is a finite set for all $\gamma \in \Gamma \mathscr{A}$, and

(ii) cofinitary (cf. [Kapranov et al. 2012]) if for every $[X] \in$ Iso $\mathscr{A}$, the set

$$
\left\{([A],[B]) \in \operatorname{Iso} \mathscr{A} \times \text { Iso } \mathscr{A}:[X] \in \underline{\operatorname{Ext}}_{\mathscr{A}}^{1}([A],[B])\right\}
$$

is finite.

Since $\mathcal{E}(M, N)_{X}$ identifies with a subset of $\operatorname{Hom}_{\mathscr{A}}(N, X) \times \operatorname{Hom}_{\mathscr{A}}(X, M)$, any profinitary category is necessarily finitary.

Proposition 2.12. Let $\mathscr{A}$ be a profinitary category. Then $\Gamma_{\mathscr{A}}$ is naturally ordered and is generated by its minimal elements.

A proof of this proposition is given in $§ 5.2$.

Remark 2.13. One can characterize profinitary categories as follows. If $\mathscr{A}$ is Homfinite and its Grothendieck monoid is locally finite, as defined before Theorem 1.4, and Ind $\mathscr{A}_{\gamma}$ is finite for all $\gamma \in \Gamma_{\mathscr{A}}$, then $\mathscr{A}$ is profinitary.

Theorem 2.14. Any profinitary abelian category has the finite length property, hence is Krull-Schmidt.

We prove this theorem in $§ 5.3$. This result, together with Theorem 1.4, yields Corollary 1.5(a). 
Remark 2.15. The finite length property in an abelian category $\mathscr{A}$ is much stronger than the Krull-Schmidt property. For instance, the Grothendieck monoid of an abelian category with the finite length property is freely generated by classes of simple objects and the canonical homomorphism $\Gamma_{\mathscr{A}} \rightarrow K_{0}(\mathscr{A})$ is injective. On the other hand, the category of coherent sheaves on $\mathbb{P}^{1}$ is Krull-Schmidt, but lacks the finite length property and each Grothendieck class Iso $\mathscr{A}_{\gamma}, \gamma \neq 0$ is infinite.

2.5. Comultiplication and primitive generation. Let $\mathscr{A}$ be any Hom-finite exact category. Define a linear map $\Delta: H_{\mathscr{A}} \rightarrow H_{\mathscr{A}} \widehat{\otimes} H_{\mathscr{A}}$ by

$$
\Delta([C])=\sum_{[A],[B] \in \mathrm{Iso} \mathscr{A}} F_{C}^{A, B} \cdot[A] \otimes[B],
$$

where $H_{\mathscr{A}} \widehat{\otimes} H_{\mathscr{A}}$ is the completion of the usual tensor product with to the $\Gamma_{\mathscr{A}}$-grading and $F_{C}^{A, B}$ is the dual Hall number given by

$$
F_{C}^{A, B}=\frac{\#\left(\mathrm{Aut}_{\mathscr{A}} A \times \mathrm{Aut}_{\mathscr{A}} B\right)}{\# \operatorname{Aut}_{\mathscr{A}} C} F_{B, A}^{C} .
$$

It follows from Riedtmann's formula [1994] that

$$
F_{C}^{A, B}=\frac{\# \operatorname{Ext}_{\mathscr{A}}^{1}(B, A)_{C}}{\# \operatorname{Hom}_{\mathscr{A}}(B, A)}
$$

where $\operatorname{Ext}_{\mathscr{A}}^{1}(B, A)_{C}=\mathcal{E}(B, A)_{C} / \operatorname{Aut}_{\mathscr{A}} C$. Also define a linear map $\varepsilon: H_{\mathscr{A}} \rightarrow \mathbb{Q}$ by

$$
\varepsilon([C])=\delta_{[0],[C]} .
$$

The following fact is obvious.

Lemma 2.16. (a) $H_{\mathscr{A}}$ is a topological coalgebra with respect to the above comultiplication and counit.

(b) If $\mathscr{A}$ is cofinitary then $H_{\mathscr{A}}$ is an ordinary coalgebra, that is, the image of the comultiplication $\Delta$ lies in $H_{\mathscr{A}} \otimes H_{\mathscr{A}}$.

For any coalgebra $C$ with unity denote by $\operatorname{Prim}(C)$ the set of all primitive elements, i.e.,

$$
\operatorname{Prim}(C)=\{c \in C: \Delta(c)=c \otimes 1+1 \otimes c\} .
$$

Definition 2.17. Let $A$ be both a unital algebra and a coalgebra over a field $\mathbb{F}$. We say that $A$ is a quasi-Nichols algebra if $A$ decomposes as $\mathbb{F} \oplus V \oplus\left(\sum_{r>1} V^{r}\right)$ where $V=\operatorname{Prim}(A)$.

The following is the main result of the paper (Main Theorem 1.2) and is proven in $§ 6.4$. 
Theorem 2.18. Let $\mathscr{A}$ be a profinitary and cofinitary exact category. Then the Hall algebra $H_{\mathscr{A}}$ is quasi-Nichols.

This theorem has the following useful corollary, which we prove in $\S 6.5$.

\section{Corollary 2.19. Let}

$$
P=\operatorname{ker} \varepsilon \cdot \operatorname{ker} \varepsilon=\mathbb{Q}\left\{[M][N]:[M],[N] \in(\text { Iso } \mathscr{A})^{+}\right\}, \quad P_{\gamma}:=P \cap\left(H_{\mathscr{A}}\right)_{\gamma}
$$

Then $P=\sum_{k \geq 2} \operatorname{Prim}\left(H_{\mathscr{A}}\right)^{k}=\sum_{k \geq 2}(\mathbb{Q} \text { Ind } \mathscr{A})^{k}$ and $\left(H_{\mathscr{A}}\right)_{\gamma}=\operatorname{Prim}\left(H_{\mathscr{A}}\right)_{\gamma} \oplus P_{\gamma}$ for all $\gamma \in \Gamma_{\mathscr{A}}^{+}$.

A natural question is to compute dimensions of $\operatorname{Prim}\left(H_{\mathscr{A}}\right)_{\gamma}, \gamma \in \Gamma_{\mathscr{A}}^{+}$. The following is a refinement of Proposition 1.8.

Proposition 2.20. In the notation (1-1) we have

$$
m_{\gamma}=\operatorname{dim}_{\mathbb{Q}}\left(P_{\gamma} \cap \mathbb{Q} \text { Ind } \mathscr{A}_{\gamma}\right)
$$

for all $\gamma \in \Gamma_{\mathscr{A}}^{+}$. In particular, if Ind $\mathscr{A}_{\gamma} \subset P_{\gamma}$ then $\operatorname{Prim}\left(H_{\mathscr{A}}\right)_{\gamma}=0$.

We prove Proposition 2.20 in $\S 6.5$, as well as the following observation, which is useful for computing primitive elements.

Lemma 2.21. Each primitive element contains at least one isomorphism class $[X] \in$ Ind $\mathscr{A}$ in its decomposition with respect to the basis Iso $\mathscr{A}$ of $H_{\mathscr{A}}$. In other words, $\operatorname{Prim}\left(H_{\mathscr{A}}\right) \cap \mathbb{Q}($ Iso $\mathscr{A} \backslash$ Ind $\mathscr{A})=\{0\}$.

2.6. Hereditary categories and Nichols algebras. Let $\Gamma$ be an abelian monoid and let $\mathscr{C}_{\Gamma}$ be the tensor category of $\Gamma$-graded vector spaces $V=\bigoplus_{\gamma \in \Gamma} V_{\gamma}$ over a field $\mathbb{F}$. The following fact can be easily checked.

Lemma 2.22. For each bicharacter $\chi: \Gamma \times \Gamma \rightarrow \mathbb{F}^{\times}$the category $\mathscr{C}_{\Gamma}$ is a braided tensor category $\left(\mathscr{C}_{\Gamma}, \Psi\right)$ with the braiding $\Psi_{U, V}: U \otimes V \rightarrow V \otimes U$ for objects $U, V$ in $\mathscr{C}_{\Gamma}$ given by

$$
\Psi_{U, V}(u \otimes v)=\chi(\gamma, \delta) v \otimes u,
$$

for any $u \in U_{\gamma}, v \in V_{\delta}, \gamma, \delta \in \Gamma$.

By a slight abuse of notation, given a bicharacter $\chi: \Gamma \times \Gamma \rightarrow \mathbb{F}^{\times}$we denote this braided tensor category $\mathscr{C}_{\Gamma}$ by $\mathscr{C}_{\chi}$.

Now let $\mathscr{A}$ be a finitary hereditary category, i.e., $\operatorname{Ext}_{\mathscr{A}}^{i}(M, N)=0$ for $i>1$ and all $M, N \in \mathscr{A}$. Let $\chi_{\mathscr{A}}: \Gamma \times \Gamma \rightarrow \mathbb{Q}^{\times}$be the bicharacter given by

$$
\chi_{\mathscr{A}}(|M|,|N|)=\frac{\# \operatorname{Ext}_{\mathscr{A}}^{1}(M, N)}{\# \operatorname{Hom}_{\mathscr{A}}(M, N)} .
$$

The bicharacter $\chi_{\mathscr{A}}$ is easily seen to be well-defined because it is just the (multiplicative) Euler form. 
Nichols algebras were formally defined in [Andruskiewitsch and Schneider 2002].

Definition 2.23 [Andruskiewitsch and Schneider 2002, Definition 2.1]. Let $(\mathscr{C}, \Psi)$ be a braided $\mathbb{F}$-linear tensor category with a braiding $\Psi$. Let $V$ be an object in $(\mathscr{C}, \Psi)$. A graded bialgebra with unity $B=\bigoplus_{n \geq 0} B_{n}$ in $(\mathscr{C}, \Psi)$ is called a Nichols algebra of $V$ if $B_{0}=\mathbb{F}, B_{1}=V$ and $B$ is generated, as an algebra, by $B_{1}=\operatorname{Prim}(B)$.

For each object $V$ of a braided tensor category $(\mathscr{C}, \Psi)$, the tensor algebra $T(V)$ is a graded bialgebra (even a Hopf algebra) in $(\mathscr{C}, \Psi)$ with the coproduct determined by requiring each $v \in V$ to be primitive and the grading defined by assigning degree 1 to elements of $V$. It is well-known [Andruskiewitsch and Schneider 2002, Proposition 2.2] that the Nichols algebra of $V$ is unique up to an isomorphism and is the quotient of $T(V)$ by the maximal graded bi-ideal $\mathfrak{I}$ of $T(V)$ which is an object in $(\mathscr{C}, \Psi)$ and satisfies $\mathfrak{I} \cap V=\{0\}$. Henceforth we denote the Nichols algebra of $V$ by $\mathcal{B}(V)$.

The following is proved in [Andruskiewitsch and Schneider 2002, Corollary 2.3].

Lemma 2.24. The assignment $V \mapsto \mathcal{B}(V)$ defines a functor from $(\mathscr{C}, \Psi)$ to the category of bialgebras in $(\mathscr{C}, \Psi)$. Moreover, for any morphism $f: U \rightarrow V$ in $(\mathscr{C}, \Psi)$, the kernel of the corresponding homomorphism $\mathcal{B}(f)$ is the (bi-)ideal in $\mathcal{B}(U)$ generated by ker $f \subset U$.

The following fact is immediate from the definitions.

Lemma 2.25. Let $B$ be a bialgebra in $(\mathscr{C}, \Psi)$ which is a quasi-Nichols algebra. Then $B$ is Nichols if and only if $\sum_{r \geq 2}(\operatorname{Prim}(B))^{r}$ is direct.

The following extends the main result of [Sevenhant and Van Den Bergh 2001]. Theorem 2.26. For any profinitary hereditary abelian category $\mathscr{A}$, the Hall algebra

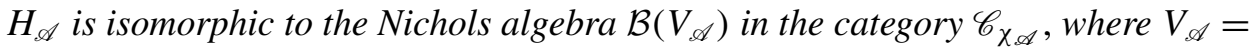
$\operatorname{Prim}\left(H_{\mathscr{A}}\right)$.

We prove this theorem in $\S 7.2$.

Remark 2.27. In fact, the original result of [Sevenhant and Van Den Bergh 2001, Theorem 1.1] follows from Theorem 2.26. The classification of diagonally braided Nichols algebras was obtained in [Andruskiewitsch and Schneider 2002, §5] and, in particular, generalizes some results of [Sevenhant and Van Den Bergh 2001].

\section{Examples}

In this section we construct primitive elements in several Hall algebras and provide supporting evidence for Conjectures 1.13 and 1.10. Throughout this section we write $\bar{\Delta}(x)=\Delta(x)-x \otimes 1-1 \otimes x$ (thus, $x$ is primitive if and only if $x \in \operatorname{ker} \bar{\Delta}$ ). Needless to say, every (almost) simple object $S$ satisfies $\bar{\Delta}([S])=0$ so we focus 
only on nonsimple primitive elements. In this section, $k$ always denotes a finite field with $q$ elements and all categories are assumed to be $\mathbb{k}$-linear.

3.1. Classical Hall-Steinitz algebra. Let $R$ be a principal ideal domain such that $R / \mathfrak{m}$ is a finite field for any maximal ideal $\mathfrak{m}$ of $R$. Let $\mathscr{A}=\mathscr{A}(\mathfrak{m})$ be the full subcategory of finite length $R$-modules $M$ satisfying $\mathfrak{m}^{r} M=0$ for some $r \geq 0$. Then for each $r>0$, there exists a unique, up to an isomorphism, indecomposable object $\mathcal{I}_{r}=R / \mathfrak{m}^{r} \in \mathscr{A}$. More generally, given a partition $\lambda=\left(\lambda_{1} \geq \cdots \geq \lambda_{k}>0\right)$, set $\mathcal{I}_{\lambda}=\mathcal{I}_{\lambda_{1}} \oplus \cdots \oplus \mathcal{I}_{\lambda_{k}}$ and write $\ell(\lambda)=k$.

Since the Euler form of $\mathscr{A}$ is identically zero and $\mathscr{A}$ is hereditary, $H_{\mathscr{A}}$ is an ordinary Hopf algebra (the braiding is trivial). The Grothendieck monoid of $\mathscr{A}$ being $\mathbb{Z}_{\geq 0}$, the algebra $H_{\mathscr{A}}$ is $\mathbb{Z}_{\geq 0}$-graded. We now provide a new (very short) proof of the following classical result.

Theorem 3.1 [Macdonald 1979; Zelevinsky 1981]. The Hall algebra $H_{\mathscr{A}}$ is commutative and cocommutative and is freely generated by the $\left[\mathcal{I}_{n}\right], n>0$. Moreover, $H_{\mathscr{A}}$ is freely generated by its primitive elements $\mathcal{P}_{n}, n>0$.

Proof. It is easy to see, using duality, that $H_{\mathscr{A}}$ is commutative, hence cocommutative. Let $\mathscr{P}$ be the set of all partitions. Given a partition $\lambda=\left(\lambda_{1} \geq \cdots \geq \lambda_{r}>0\right) \in \mathscr{P}$, let $M_{\lambda}=\left[\mathcal{I}_{\lambda_{1}}\right] \cdots\left[\mathcal{I}_{\lambda_{r}}\right]$. By Theorem 2.4, the set $\left\{M_{\lambda}\right\}_{\lambda \in \mathscr{P}}$ is a basis of $H_{\mathscr{A}}$, hence $H_{\mathscr{A}}$ is freely generated by the isomorphism classes of indecomposables $\left[\mathcal{I}_{n}\right], n>0$. Since $H_{\mathscr{A}}$ is commutative, $P=\operatorname{ker} \varepsilon \cdot \operatorname{ker} \varepsilon$ is spanned by the $M_{\lambda}$ with $\ell(\lambda) \geq 2$, hence $\mathbb{Q}$ Ind $\mathscr{A} \cap P=\{0\}$ and by Proposition $2.20, \operatorname{dim}_{\mathbb{Q}} \operatorname{Prim}\left(H_{\mathscr{A}}\right)_{n}=\#$ Ind $\mathscr{A}_{n}=1$ for all $n>0$. Thus, for each $n>0$ we have a unique, up to a scalar, nonzero primitive element $\mathcal{P}_{n}$ in $\left(H_{\mathscr{A}}\right)_{n}$. The dimension considerations and Theorem 2.18 immediately imply that $H_{\mathscr{A}}$ is freely generated by the $\mathcal{P}_{n}, n>0$.

This theorem has the following nice corollary.

Corollary 3.2. For all $n>0$, let $x_{n} \in\left(H_{\mathscr{A}}\right)_{n} \backslash \mathbb{Q}\left(\right.$ Iso $\mathscr{A}_{n} \backslash$ Ind $\left.\mathscr{A}_{n}\right)$. Then $\left\{x_{n}\right\}_{n>0}$ freely generates $H_{\mathscr{A}}$. In particular, $E_{\mathscr{A}}=H_{\mathscr{A}}$.

The elements $\mathcal{P}_{n}$ can be computed explicitly (see, e.g., [Hubery 2005, §5]), namely

$$
\mathcal{P}_{n}=\sum_{\lambda \vdash n}\left(\prod_{j=1}^{\ell(\lambda)-1}\left(1-q^{j}\right)\right)\left[\mathcal{I}_{\lambda}\right]
$$

where $q=|R / \mathfrak{m}|$.

Under the isomorphism $\psi: H_{\mathscr{A}} \rightarrow \operatorname{Sym},\left[\mathcal{I}_{\lambda}\right] \mapsto q^{-n(\lambda)} P_{\lambda}\left(x ; q^{-1}\right)$ [Macdonald 1979; Zelevinsky 1981], where Sym is the algebra of symmetric polynomials in infinitely many variables and $P_{\lambda}(x ; t)$ is the Hall-Littlewood polynomial, the image of $\mathcal{P}_{n}$ is the $n$-th power sum $p_{n}$. As shown in [Zelevinsky 1981], the $p_{n}$ are primitive 
elements in Sym with the comultiplication defined by

$$
\Delta\left(e_{n}\right)=\sum_{i=0}^{n} e_{i} \otimes e_{n-i},
$$

where $e_{r}$ is the $r$-th elementary symmetric polynomial, which equals $q^{-\left(\begin{array}{c}r \\ 2\end{array}\right)} \psi\left(\left[\mathcal{I}_{\left(1^{r}\right)}\right]\right)$. Note also that $\psi\left(\sum_{\lambda \vdash n}\left[\mathcal{I}_{\lambda}\right]\right)$ is the $n$-th complete symmetric function $h_{n}$.

Since $C_{\mathscr{A}}=\mathbb{Q}\left[\mathcal{P}_{1}\right]$, we have $C_{\mathscr{A}} \subsetneq H_{\mathscr{A}}$. Since $\operatorname{dim}_{\mathbb{Q}} \operatorname{Prim}\left(H_{\mathscr{A}}\right)_{n}=1$ for all $n>0$, it follows that $U_{\mathscr{A}}=H_{\mathscr{A}}$. Thus, $C_{\mathscr{A}} \subsetneq E_{\mathscr{A}}=U_{\mathscr{A}}=H_{\mathscr{A}}$.

3.2. Homogeneous tubes. Let $\mathscr{A}$ be the category of finite dimensional $\mathbb{k}$-representations of a tame acyclic quiver $Q$. Then $\mathscr{A}$ decomposes into a triple of subcategories of preprojective, preinjective and regular representations (see [Auslander et al. 1995, Chapter VIII]) which we denote, as in [Berenstein and Greenstein 2013, §5], by $\mathscr{A}_{-}, \mathscr{A}_{+}$and $\mathscr{A}_{0}$, respectively. The category $\mathscr{A}_{0}$ can be further decomposed into the so-called stable tubes, that is, components of the Auslander-Reiten quiver of $\mathscr{A}$ on which the Auslander translation acts as an autoequivalence of finite order, called the rank of the tube. It is well-known that rank 1, or homogeneous, tubes are parametrized by the set $\mathbb{k} \mathbb{P}^{1}$ of homogeneous prime ideals in $\mathbb{k}[x, y]$. Given a homogeneous prime ideal $\rho$, let $\operatorname{deg} \rho$ be the degree of a generator of that ideal and denote by $\mathscr{T}_{\rho}$ the corresponding rank 1 tube. Then $\mathscr{T}_{\rho}$ is equivalent to the category of nilpotent representations of $\mathbb{K}[x]$ where $[\mathbb{K}: \mathbb{k}]=\operatorname{deg} \rho$ and its Hall algebra is isomorphic to the classical Hall-Steinitz algebra. Thus, for each $r>0, \mathscr{T}_{\rho}$ contains a unique indecomposable $\mathcal{I}_{r}(\rho)$ of length $r$. Given a partition $\lambda=\left(\lambda_{1} \geq \cdots \geq \lambda_{k}>0\right)$, let $\mathcal{I}_{\lambda}(\rho)=\mathcal{I}_{\lambda_{1}}(\rho) \oplus \cdots \oplus \mathcal{I}_{\lambda_{k}}(\rho)$. By $\S 3.1$ the elements

$$
\mathcal{P}_{n}(\rho)=\sum_{\lambda \vdash n}\left(\prod_{j=1}^{\ell(\lambda)-1}\left(1-q^{j \operatorname{deg} \rho}\right)\right)\left[\mathcal{I}_{\lambda}(\rho)\right]
$$

are primitive in $H_{\mathscr{T}_{\rho}}$. Let $\mathscr{A}_{0, h}$ be the full subcategory of homogeneous objects in $\mathscr{A}_{0}$ (cf. [Dlab and Ringel 1976, Theorem 3.5]). Since $H_{\mathscr{A}_{0, h}}$ is isomorphic to the tensor product of the $H_{\mathscr{P}_{\rho}}$ as a bialgebra, this gives all primitive elements in $H_{\mathscr{A}_{0, h}}$. The Grothendieck monoid of $\mathscr{A}_{0, h}$ equals the direct sum of infinitely many copies (indexed by $\rho \in \mathbb{k} \mathbb{P}^{1}$ ) of $\mathbb{Z}_{\geq 0}$.

However, the elements $\mathcal{P}_{n}(\rho)$ are not primitive in $H_{\mathscr{A}}$ since an object in $\mathscr{A}_{0, h}$ can have preprojective subobjects and preinjective quotients. They can be used to construct primitive elements in $H_{\mathscr{A}}$.

Conjecture 3.3. The elements

$$
\mathcal{P}_{n}(\rho)-\frac{1}{N(\operatorname{deg} \rho)} \sum_{\rho^{\prime} \in \mathbb{R} \mathbb{P}^{1}: \operatorname{deg} \rho^{\prime}=\operatorname{deg} \rho} \mathcal{P}_{n}\left(\rho^{\prime}\right),
$$


are primitive in $H_{\mathscr{A}}$, where $N(d)$ is the number of elements of $\mathbb{k P}^{1}$ of degreed (that is, $N(1)=|\mathbb{k}|+1$ while $N(d), d>1$, is the number of irreducible monic polynomials of degree $d$ in one variable).

This formula can be easily checked in small cases (see, for example, §3.8) or for the Kronecker quiver, using the results of [Szántó 2006]. Since $F_{M}^{I, P}=0$ for all $P \in \mathscr{A}_{-}$and $I \in \mathscr{A}_{+}$, the above conjecture is an immediate consequence of the next conjecture.

Conjecture 3.4. ${ }^{1}$ Let $I \in \mathscr{A}_{+}$and $P \in \mathscr{A}_{-}$. Then for any partition $\lambda$ we have $F_{\mathcal{I}_{\lambda}(\rho)}^{P, I}=F_{\mathcal{I}_{\lambda}\left(\rho^{\prime}\right)}^{P, I}$ where $\rho, \rho^{\prime} \in \mathbb{k} \mathbb{P}^{1}$ with $\operatorname{deg} \rho=\operatorname{deg} \rho^{\prime}$.

This is known to hold in some special cases (see for example [Szántó 2006; Hubery 2004]).

In the category $\mathscr{A}$, we have $C_{\mathscr{A}}=E_{\mathscr{A}}=U_{\mathscr{A}} \subsetneq H_{\mathscr{A}}$. On the other hand, for $\mathscr{A}_{0}$ we have $C_{\mathscr{A}_{0}} \subsetneq E_{\mathscr{A}_{0}}=U_{\mathscr{A}_{0}}=H_{\mathscr{A}_{0}}$ and similarly for each homogeneous tube.

3.3. A tame valued quiver. Consider now the valued quiver $1 \stackrel{(4,1)}{\longrightarrow} 2$. Let $\mathbb{k}_{2}$ be a field extension of $\mathbb{k}_{1}=\mathbb{k}_{k}$ of degree 4 . Note that $\mathbb{k}_{2}$ contains precisely $q^{4}-q^{2}$ elements of degree 4 over $\mathbb{k}$ and $q^{2}-q$ elements of degree 2 . A representation of this quiver is a triple $\left(V_{1}, V_{2}, f\right)$ where $V_{i}$ is a $\mathbb{k}_{i}$-vector space and $f \in \operatorname{Hom}_{\mathbb{k}}\left(V_{1}, V_{2}\right)$. Finally, a morphism $\left(V_{1}, V_{2}, f\right) \rightarrow\left(W_{1}, W_{2}, g\right)$ is a pair $\left(\varphi_{1}, \varphi_{2}\right)$ where $\varphi_{i} \in \operatorname{Hom}_{\mathbb{k}_{i}}\left(V_{i}, W_{i}\right)$ and $g \circ \varphi_{1}=\varphi_{2} \circ g$.

The smallest indecomposable regular representation is $\left(\mathbb{k}_{1}^{2}, \mathbb{k}_{2}, f\right)$, where $f$ is injective. Thus, $f$ is given by a pair $(\lambda, \mu) \in \mathbb{k}_{2} \times \mathbb{k}_{2}$ which is linearly independent over $k$ (this pair is the image under $f$ of the standard basis of $k_{1}^{2}$ ). It is easy to see that, up to an isomorphism, such a pair can be assumed to be of the form $(\lambda, 1)$ where $\lambda \in \mathbb{k}_{2} \backslash \mathbb{k}_{1}$. Denote the resulting representation by $E_{1}(\lambda)$. A morphism $f: E_{1}(\lambda) \rightarrow E_{1}\left(\lambda^{\prime}\right)$ is uniquely determined by a matrix $\varphi_{1}=\left(\begin{array}{ll}a & b \\ c & d\end{array}\right) \in M_{2}(\mathbb{k})$ and $\varphi_{2} \in \mathbb{k}_{2}$ and we have

$$
\left(b \lambda^{\prime}+d\right) \lambda=a \lambda^{\prime}+c .
$$

If $\lambda$ has degree 4 over $\mathbb{k}$ then $\operatorname{End}_{\mathscr{A}} E_{1}(\lambda) \cong \mathbb{k}$ and Aut $E_{\mathscr{A}} E_{1}(\lambda) \cong \mathbb{k}^{\times}$. Otherwise, $\operatorname{End}_{\mathscr{A}} E_{1}(\lambda) \cong L$ and $\operatorname{Aut}_{\mathscr{A}} E_{1}(\lambda) \cong L^{\times}$where $[L: \mathbb{k}]=2$. It follows that all $E_{1}(\lambda)$ with $\operatorname{deg}_{\mathbb{k}} \lambda=2$ are isomorphic, since the stabilizer of such a $\lambda$ in $\operatorname{GL}(2, \mathbb{k})$ has index $q^{2}-q$, and that there are $q$ nonisomorphic representations $E_{1}(\lambda)$ with $\operatorname{deg}_{\mathbb{k}} \lambda=4$. It is easy to see that for any $\lambda \in \mathbb{k}_{2} \backslash \mathbb{k}_{1}$ we have $\left(q^{2}-1\right)(q-1)$ short exact sequences

$$
0 \rightarrow P_{1} \rightarrow E_{1}(\lambda) \rightarrow S_{1} \rightarrow 0
$$

${ }^{1}$ After the present paper was accepted for publication, we were informed that a proof of Conjecture 3.4 was announced in [Deng and Ruan 2015]. 
and $q\left(q^{4}-1\right)\left(q^{2}-1\right)(q-1)$ short exact sequences

$$
0 \rightarrow S_{2} \rightarrow E_{1}(\lambda) \rightarrow S_{1}^{\oplus 2} \rightarrow 0 .
$$

As a result, we conclude that

$$
\bar{\Delta}\left(E_{1}(\lambda)\right)=\frac{\left(q^{2}-1\right)(q-1)}{\left|\operatorname{Aut}_{\mathscr{A}} E_{1}(\lambda)\right|}\left(\left[P_{1}\right] \otimes\left[S_{1}\right]+q\left(q^{4}-1\right)\left[S_{2}\right] \otimes\left[S_{1}^{\oplus 2}\right]\right),
$$

hence

$$
\mathcal{P}_{1}(\lambda):=E_{1}(\lambda)-\frac{1}{(q+1)\left|\operatorname{Aut}_{\mathscr{A}} E_{1}(\lambda)\right|} \sum_{\mu \in\left(\mathbb{k}_{2} \backslash \mathbb{k}_{1}\right) / \mathrm{GL}(2, \mathrm{k})}\left|\operatorname{Aut}_{\mathscr{A}} E_{1}(\mu)\right| E_{1}(\mu)
$$

is primitive, and these are all primitive elements of degree $2 \alpha_{1}+\alpha_{2}$ in $H_{\mathscr{A}}$. There is precisely one linear relation among them, namely

$$
\sum_{\lambda \in\left(\mathbb{k}_{2} \backslash \mathbb{k}_{1}\right) / \mathrm{GL}(2, \mathbb{k})}\left|\operatorname{Aut}_{\mathscr{A}} E_{1}(\lambda)\right| \mathcal{P}_{1}(\lambda)=0 .
$$

In this case, like in $\S 3.2, C_{\mathscr{A}}=E_{\mathscr{A}}=U_{\mathscr{A}} \subsetneq H_{\mathscr{A}}$ which supports Conjecture 1.13. Also, $\operatorname{dim}_{\mathbb{Q}} \operatorname{Prim}\left(H_{\mathscr{A}}\right)_{2 \alpha_{1}+\alpha_{2}}=q$ and $m_{2 \alpha_{1}+\alpha_{2}}=1$.

3.4. Hereditary categories defined by submonoids. The next two examples are special cases of the following construction. Consider a submonoid $\Gamma_{0}$ of the Grothendieck monoid $\Gamma$ of an abelian category $\mathscr{A}$, and define a full subcategory $\mathscr{A}\left(\Gamma_{0}\right)$ of $\mathscr{A}$ whose objects $X$ satisfy $|X| \in \Gamma_{0}$. By construction, $\mathscr{A}\left(\Gamma_{0}\right)$ is closed under extensions and hence is exact.

First, let $\mathscr{A}$ be the category of $\mathbb{k}$-representations of the quiver $1 \rightarrow 2$. Then $\Gamma_{\mathscr{A}}$ is freely generated by $\alpha_{i}=\left|S_{i}\right|$ where the $S_{i}, i=1,2$ are simple objects. Fix $r>0$. Let $\Gamma_{r}=\mathbb{Z}_{\geq 0}\left(\alpha_{1}+r \alpha_{2}\right)$ and set $\mathscr{B}_{r}=\mathscr{A}\left(\Gamma_{r}\right)$. Let $P_{1}=I_{2}$ be the projective cover of $S_{1}$ and the injective envelope of $S_{2}$ in $\mathscr{A}$. Then in $H_{\mathscr{A}}$ we have

$$
\left[S_{1}\right]\left[S_{2}\right]=\left[S_{2}\right]\left[S_{1}\right]+\left[P_{1}\right], \quad\left[S_{1}\right]\left[P_{1}\right]=q\left[P_{1}\right]\left[S_{1}\right], \quad\left[P_{1}\right]\left[S_{2}\right]=q\left[S_{2}\right]\left[P_{1}\right] .
$$

Every object in $\mathscr{B}_{r}$ is isomorphic to $S_{1}^{\oplus a} \oplus P_{1}^{\oplus b} \oplus S_{2}^{\oplus(r a+(r-1) b)}, a, b \geq 0$. The only simple objects in $\mathscr{B}_{r}$, up to an isomorphism, are $X_{1}=S_{1} \oplus S_{2}^{\oplus r}$ and $X_{2}=S_{2}^{\oplus r-1} \oplus P_{1}$. Then $\left[X_{1}\right]$ is a nonzero multiple of $E_{1}=\left[S_{2}\right]^{r}\left[S_{1}\right]$, and $\left[X_{2}\right]$ of $E_{2}=\left[S_{2}\right]^{r-1}\left[P_{1}\right]$. In particular, the $E_{i}$ are primitive elements of $H_{\mathscr{B}_{r}}$. Using (3-1) we can show that $E_{1}$ and $E_{2}$ satisfy the relation

$$
E_{2} E_{1}=q^{r-1} E_{1} E_{2}-[r-1]_{q} E_{2}^{2},
$$

where $[s]_{q}=1+\cdots+q^{s-1}$. The Grothendieck monoid of $\mathscr{B}_{r}$ is generated by $\beta_{i}=\left|X_{i}\right|, i=1,2$, subject to the relation $\beta_{1}+\beta_{2}=2 \beta_{1}=2 \beta_{2}$ (thus $\Gamma_{\mathscr{B}_{r}}$ does not coincide with $\Gamma_{r}$ and is not even a submonoid of $\Gamma_{\mathscr{A}}$ ). It is not hard to check that $E_{1}$ and $E_{2}$ generate $H_{\mathscr{B}_{r}}$ and hence form a basis of $\operatorname{Prim}\left(H_{\mathscr{B}_{r}}\right)$. 
In this case we have $C_{\mathscr{B}_{r}}=U_{\mathscr{B}_{r}}=E_{\mathscr{B}_{r}}=H_{\mathscr{B}_{r}}$ and so Conjecture 1.13 holds.

A more complicated example is obtained as follows. Let $\mathscr{A}$ be the category of $\mathbb{k}$-representations of the quiver $1 \rightarrow 0 \leftarrow 2$. As in the previous example, $\Gamma_{\mathscr{A}}$ is freely generated by $\alpha_{i}=\left|S_{i}\right|, 0 \leq i \leq 2$. Let $\Gamma_{\circ}=\left\{s \alpha_{0}+r \alpha_{1}+r \alpha_{2}: r, s \in \mathbb{Z}_{\geq 0}\right\}$ and let $\mathscr{B}=\mathscr{A}\left(\Gamma_{\circ}\right)$. Let $P_{i}$ be the projective cover of $S_{i}$ in $\mathscr{A}$ and $I_{i}$ be its injective envelope. Thus, $I_{1}=S_{1}, I_{2}=S_{2},\left|I_{0}\right|=\alpha_{0}+\alpha_{1}+\alpha_{2},\left|P_{1}\right|=\alpha_{0}+\alpha_{1}, P_{0}=S_{0}$ and $\left|P_{2}\right|=\alpha_{2}+\alpha_{0}$. The simple objects in $\mathscr{B}$ are $S_{1} \oplus S_{2}$ and $S_{0}$, while the nonsimple indecomposable objects are

$$
P_{1} \oplus S_{2}, \quad P_{2} \oplus S_{1}, \quad P_{1} \oplus P_{2}, \quad I_{0} .
$$

The Grothendieck monoid of $\mathscr{B}$ is freely generated by $\beta_{1}=\left|S_{1} \oplus S_{2}\right|$ and $\beta_{0}=\left|S_{0}\right|$. Clearly, $Y_{1}=\left[S_{1} \oplus S_{2}\right]$ and $Y_{0}=\left[S_{0}\right]$ are primitive in $H_{\mathscr{B}}$. We also have two linearly independent primitive elements of degree $\beta_{1}+\beta_{0}$, say

$$
\begin{aligned}
& Z_{1}=\left[I_{0}\right]-(q-1)\left[P_{1} \oplus S_{2}\right], \\
& Z_{2}=\left[I_{0}\right]-(q-1)\left[P_{2} \oplus S_{1}\right] .
\end{aligned}
$$

Then

$$
\left[Z_{1}, Z_{2}\right]=0, \quad\left[Y_{1}, Z_{1}\right]_{q}=\left[Y_{1}, Z_{2}\right]_{q}=0, \quad\left[Z_{1}, Y_{0}\right]_{q}=\left[Z_{2}, Y_{0}\right]_{q}=0,
$$

and

$$
\left.\left[Y_{1},\left[Y_{1}, Y_{0}\right]\right]_{q^{2}}=Y_{1}\left(Z_{1}+Z_{2}\right), \quad\left[\left[Y_{1}, Y_{0}\right], Y_{0}\right]_{q}, Y_{0}\right]_{q^{2}}=0,
$$

where $[a, b]_{t}=a b-t b a$. Here $C_{\mathscr{B}}=E_{\mathscr{B}}=U_{\mathscr{B}} \subsetneq H_{\mathscr{B}}$ which again supports Conjecture 1.13. Also, we have a unique imaginary simple root $\beta_{1}+\beta_{0}$, and $\operatorname{dim}_{\mathbb{Q}} \operatorname{Prim}\left(H_{\mathscr{B}}\right)_{\beta_{1}+\beta_{0}}=2$ while $m_{\beta_{1}+\beta_{0}}=1$.

3.5. Sheaves on projective curves. Consider the category $\mathscr{A}$ of coherent sheaves on $\mathbb{P}^{1}(\mathbb{k})$ (cf. [Burban and Schiffmann 2012; Kapranov 1997; Baumann and Kassel 2001]). Following [Baumann and Kassel 2001], $\mathscr{A}$ is equivalent to the category with objects $\left(M^{\prime}, M^{\prime \prime}, \phi\right)$ where $M^{\prime}$ is a $\mathbb{k}[z]$-module, $M^{\prime \prime}$ is a $\mathbb{k}\left[z^{-1}\right]$-module and $\phi$ is an isomorphism of $\mathbb{k}\left[z, z^{-1}\right]$-modules $M_{z}^{\prime} \rightarrow M_{z^{-1}}^{\prime \prime}$. In particular, for any $n \in \mathbb{Z}$, we have an indecomposable object $\mathcal{O}(n)=\left(\mathbb{k}[z], \mathbb{k}\left[z^{-1}\right], \phi_{n}\right)$ where $\phi_{n} \in$ Aut $\mathbb{k}\left[z, z^{-1}\right]$ is multiplication by $z^{-n}$. We have (cf. [Baumann and Kassel 2001])

$$
\operatorname{dim}_{\mathbb{k}} \operatorname{Hom}_{\mathscr{A}}(\mathcal{O}(m), \mathcal{O}(n))=\max (0, n-m+1)
$$

and any nonzero morphism $\mathcal{O}(m) \rightarrow \mathcal{O}(n)$ is injective.

Consider now the full subcategory $\mathscr{A}_{\text {lc }}$ of locally free coherent sheaves on $\mathbb{P}^{1}$. Any object in $\mathscr{A}_{\mathrm{lc}}$ is isomorphic to a direct sum of objects of the form $\mathcal{O}(\mathrm{m})$ and these are precisely the indecomposables in $\mathscr{A}_{\mathrm{lc}}$. The Grothendieck monoid of $\mathscr{A}_{\mathrm{lc}}$ identifies with $\{(0,0)\} \cup \mathbb{Z}_{\geq 0} \times \mathbb{Z}$ with $|\mathcal{O}(n)|=(1, n)$. Note that $\mathscr{A}$ lc has no simple objects. The category $\mathscr{A}_{\mathrm{c}}$ is closed under extensions and hence is exact. Since 
$\mathcal{A}_{\mathrm{lc}}$ is Krull-Schmidt, its Hall algebra has a basis consisting of ordered monomials on $X_{m}:=[\mathcal{O}(m)]$ for any total order on $\mathbb{Z}$. Since $m<n$ implies that $\mathcal{O}(n) / \mathcal{O}(m)$ is not an object in $\mathscr{A}_{\mathrm{lc}}$, it follows that $\mathcal{O}(m)$ is almost simple, hence $X_{m}$ is primitive for all $m \in \mathbb{Z}$. Thus, $H_{\mathscr{A} / \mathrm{c}}$ is primitively generated. By [Baumann and Kassel 2001, Theorem 10(iii)] the defining relations in $H_{\mathscr{A}_{1 \mathrm{c}}}$ are

$$
X_{n} X_{m}=q^{n-m+1} X_{m} X_{n}+\left(q^{2}-1\right) q^{n-m-1} \sum_{a=1}^{\lfloor(n-m) / 2\rfloor} X_{m+a} X_{n-a}, \quad m<n .
$$

However, Theorem 2.18 does not apply to the Hall algebra of $\mathscr{A}$ or $\mathscr{A}_{\mathrm{lc}}$ since the categories $\mathscr{A}$ or even $\mathscr{A}_{\text {lc }}$ are neither profinitary nor cofinitary. For example, every object $\mathcal{O}(m) \oplus \mathcal{O}(n), m>n$ appears as the middle term of a short exact sequence

$$
0 \rightarrow \mathcal{O}(n-a) \rightarrow \mathcal{O}(m) \oplus \mathcal{O}(n) \rightarrow \mathcal{O}(m+a) \rightarrow 0
$$

for all $a \geq 0$.

On the other hand, the Hall algebra of the subcategory of torsion sheaves is isomorphic to the Hall algebra of the regular subcategory for the valued quiver $1 \stackrel{(2,2)}{\longrightarrow} 2$, or, equivalently, the Kronecker quiver.

It should be noted that the Hall algebra of the subcategory of preprojective modules $\mathscr{B}_{+}$in the category $\mathscr{B}$ of $\mathbb{k}$-representations of the Kronecker quiver is isomorphic to the subalgebra of $H_{\mathscr{A} / \mathrm{c}}$ generated by the $X_{m}$ for $m>0$. Indeed, $\Gamma_{\mathscr{B}_{+}} \cong \mathbb{Z}_{\geq 0}$, and for each $k>0$ there is a unique preprojective indecomposable $Q_{k}$ with $\left|Q_{k}\right|=k$. It is easy to see, by grading considerations, that $Q_{k}$ is primitive. Then the $\left[Q_{k}\right], k \geq 0$ can be shown to satisfy exactly the same relations as the $X_{n}$ (see [Szántó 2006, Theorem 4.2]). In this case we have

$$
C_{\mathscr{B}_{+}} \subsetneq U_{\mathscr{B}_{+}}=E_{\mathscr{B}_{+}}=H_{\mathscr{B}_{+}} .
$$

This situation can be generalized as follows. Let $X$ be a smooth projective curve and let $\mathscr{A}$ be the category of coherent sheaves on $X$. Let $\mathscr{A}_{\mathrm{lc}}^{\geq d}$ be the full subcategory of $\mathscr{A}$ whose objects are locally free sheaves of positive rank and of degree $\geq d$. Since the rank and the degree are additive on short exact sequences, this subcategory is closed under extensions. Since for a coherent sheaf $\mathcal{F}$ the possible degrees of its subsheaves of rank $r$ are bounded above (cf. [Kapranov et al. 2012, Proposition 2.5]), for any fixed pair $(r, d)$ there are finitely many subsheaves of $\mathcal{F}$ of rank $r$ and degree $d$. We conclude that the category $\mathscr{A}_{\mathrm{lc}}^{\geq d}$ is cofinitary and profinitary, hence Theorem 2.18 applies and the Hall algebra of $\mathscr{A}_{\mathrm{lc}}^{\geq d}$ is generated by its primitive elements. Results on primitive elements in this algebra can be found in [Kapranov et al. 2012, §3.2]. Note that $\mathscr{A}_{1 \mathrm{c}}$ is Krull-Schmidt, hence its Hall algebra is PBW on indecomposables. 
3.6. Nonhereditary categories of finite type. Let $\mathscr{A}$ be the category of $\mathbb{k}$-representations of the quiver

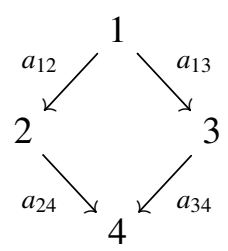

satisfying the relation $a_{24} a_{12}=0$. This category has 14 isomorphism classes of indecomposable objects, 12 of them having different images in $\Gamma_{\mathscr{A}}$ and the two remaining ones, namely the projective cover $P_{1}$ of $S_{1}$ and the injective envelope $I_{4}$ of $S_{4}$, having the same image $\alpha_{1}+\alpha_{2}+\alpha_{3}+\alpha_{4}$ (as before, $\alpha_{i}=\left|S_{i}\right|$ ).

Let $S_{i j}$ and $S_{i j k}$ be the unique, up to an isomorphism, indecomposables with $|X|=\alpha_{i}+\alpha_{j}$ and $|X|=\alpha_{i}+\alpha_{j}+\alpha_{k}$, respectively. Then $\left[S_{i j}\right],\left[S_{i j k}\right] \in P$ follows easily, hence $\operatorname{Prim}\left(H_{\mathscr{A}}\right)_{\alpha_{i}+\alpha_{j}}=0=\operatorname{Prim}\left(H_{\mathscr{A}}\right)_{\alpha_{i}+\alpha_{j}+\alpha_{k}}$ by Proposition 2.20. Let us show that $\operatorname{Prim}\left(H_{\mathscr{A}}\right)_{\alpha_{1}+\alpha_{2}+\alpha_{3}+\alpha_{4}}=0$; then the only primitive elements are those in $\operatorname{Prim}\left(H_{\mathscr{A}}\right)_{\alpha_{i}}, 1 \leq i \leq 4$.

For every object $M$ with $|M|=\alpha_{1}+\alpha_{2}+\alpha_{3}+\alpha_{4}$, except $P_{1}, I_{4}$ and $S_{2} \oplus S_{134}$, there exists a pair of objects $A, B$ such that $F_{N}^{A, B}=0$ unless $[N]=[M]$. This implies that $\operatorname{Prim}\left(H_{\mathscr{A}}\right)_{\alpha_{1}+\alpha_{2}+\alpha_{3}+\alpha_{4}}$ is contained in the linear span of [ $\left.P_{1}\right],\left[I_{4}\right]$ and $\left[S_{2} \oplus S_{134}\right]$. We have (with $h=\left|\mathbb{k}^{\times}\right|=q-1$ )

$$
\begin{aligned}
& \bar{\Delta}\left(\left[S_{2} \oplus S_{134}\right]\right)=[\left.S_{134}\right] \otimes\left[S_{2}\right]+\left[S_{2}\right] \otimes\left[S_{134}\right] \\
&+ h\left(\left[S_{2} \oplus S_{34}\right] \otimes\left[S_{1}\right]+\left[S_{2} \oplus S_{4}\right] \otimes\left[S_{13}\right]\right. \\
&\left.+\left[S_{34}\right] \otimes\left[S_{1} \oplus S_{2}\right]+\left[S_{4}\right] \otimes\left[S_{2} \oplus S_{13}\right]\right), \\
& \bar{\Delta}\left(\left[I_{4}\right]\right)=h\left(\left[S_{134}\right] \otimes\left[S_{2}\right]+\left[S_{234}\right] \otimes\left[S_{1}\right]+\left[S_{24}\right] \otimes\left[S_{13}\right]\right)+h^{2}\left(\left[S_{34}\right] \otimes\left[S_{1} \oplus S_{2}\right]+\left[S_{4}\right] \otimes\left[S_{2} \oplus S_{13}\right]\right), \\
& \bar{\Delta}\left(\left[P_{1}\right]\right)=h\left(\left[S_{34}\right] \otimes\left[S_{12}\right]+\left[S_{2}\right] \otimes\left[S_{134}\right]+\left[S_{4}\right] \otimes\left[S_{123}\right]\right) \\
&+h^{2}\left(\left[S_{2} \oplus S_{34}\right] \otimes\left[S_{1}\right]+\left[S_{2} \oplus S_{4}\right] \otimes\left[S_{13}\right]\right) .
\end{aligned}
$$

It is now clear that $\operatorname{Prim}\left(H_{\mathscr{A}}\right)_{\alpha_{1}+\alpha_{2}+\alpha_{3}+\alpha_{4}}=0$.

Let $E_{i}=\left[S_{i}\right], 1 \leq i \leq 4$. To write a presentation of $H_{\mathscr{A}}$, it is useful to introduce $Z=\left[P_{1}\right]+\left[I_{4}\right]-(q-1)\left[S_{2} \oplus S_{134}\right]$. We obtain

$$
\begin{aligned}
{\left[E_{i},\left[E_{i}, E_{j}\right]\right]_{q}=0=} & {\left[\left[E_{i}, E_{j}\right], E_{j}\right]_{q} } \\
& \text { for }(i, j) \in\{(1,2),(1,3),(2,4),(3,4)\},
\end{aligned}
$$

$$
\left[E_{2}, E_{3}\right]=0=\left[E_{1}, E_{4}\right] \text {, }
$$


and also

$$
\left[E_{4},\left[E_{1}, E_{2}\right]\right]=0, \quad\left[E_{1}, Z\right]_{q}=0=\left[Z, E_{4}\right]_{q}, \quad\left[E_{2}, Z\right]=0=\left[E_{3}, Z\right],
$$

where

$$
Z=\left[E_{1},\left[E_{2},\left[E_{3}, E_{4}\right]\right]_{q}\right]-\left[E_{4},\left[E_{3},\left[E_{2}, E_{1}\right]\right]_{q}\right] .
$$

If we consider the category of representations of the same quiver satisfying the relation $a_{24} a_{12}=a_{34} a_{13}$, its Hall algebra's subspace of primitive elements is spanned by the $E_{i}, 1 \leq i \leq 4$ which satisfy (3-2), as well as

$$
\begin{aligned}
{\left[E_{4},\left[E_{1}, E_{2}\right]\right] } & =0=\left[E_{4},\left[E_{1}, E_{3}\right]\right] \\
{\left[E_{1},\left[E_{2},\left[E_{3}, E_{4}\right]\right]\right] } & =\left[E_{4},\left[E_{3},\left[E_{1}, E_{2}\right]\right]\right] \\
& =\left[E_{4},\left[E_{2},\left[E_{1}, E_{3}\right]\right]\right]=\left[E_{1},\left[E_{3},\left[E_{2}, E_{4}\right]\right]\right] .
\end{aligned}
$$

In both cases $C_{\mathscr{A}}=E_{\mathscr{A}}=U_{\mathscr{A}}=H_{\mathscr{A}}$.

3.7. Special pairs of objects and primitive elements. Before we consider the next group of examples, we make the following observation. Suppose that we have a pair of indecomposable objects $X \not Y Y$ in $\mathscr{A}$ satisfying $\operatorname{Hom}_{\mathscr{A}}(X, Y)=0=\operatorname{Hom}_{\mathscr{A}}(Y, X)$, $\operatorname{End}_{\mathscr{A}} X \cong \operatorname{End}_{\mathscr{A}} Y \cong \mathbb{k}$ is a field and

$$
\operatorname{dim}_{\mathbb{k}} \operatorname{Ext}_{\mathscr{A}}^{1}(X, Y)=\operatorname{dim}_{\mathbb{k}} \operatorname{Ext}_{\mathscr{A}}^{1}(Y, X)=1 .
$$

Then there exist unique $\left[Z_{Y X}\right],\left[Z_{X Y}\right] \in$ Iso $\mathscr{A}$ such that

$$
\underline{\operatorname{Ext}}_{\mathscr{A}}^{1}(X, Y)=\left\{[X \oplus Y],\left[Z_{X Y}\right]\right\}, \quad \underline{\operatorname{Ext}}_{\mathscr{A}}^{1}(Y, X)=\left\{[X \oplus Y],\left[Z_{Y X}\right]\right\} .
$$

Let $\mathscr{B}=\mathscr{A}(X, Y)$ be the minimal additive full subcategory of $\mathscr{A}$ containing $X$ and $Y$ and closed under extensions. Then in $H_{\mathscr{B}}$ we have

$$
\begin{aligned}
\bar{\Delta}\left(\left[Z_{Y X}\right]\right) & =(q-1)[X] \otimes[Y], \\
\bar{\Delta}\left(\left[Z_{X Y}\right]\right) & =(q-1)[Y] \otimes[X], \\
\bar{\Delta}([X \oplus Y]) & =[X] \otimes[Y]+[Y] \otimes[X],
\end{aligned}
$$

and so

$$
\left[Z_{X Y}\right]+\left[Z_{Y X}\right]-(q-1)[X \oplus Y]
$$

is primitive in $H_{\mathscr{B}}$. Indeed, $\left|\operatorname{Ext}_{\mathscr{A}}^{1}(Y, X)_{Z_{Y X}}\right|=q-1=\left|\operatorname{Ext}_{\mathscr{A}}^{1}(X, Y)_{Z_{X Y}}\right|$ and so by Riedtmann's formula,

$$
F_{Z_{Y X}}^{X, Y}=q-1=F_{Z_{X Y}}^{Y, X}, \quad F_{X \oplus Y}^{X, Y}=F_{X \oplus Y}^{Y, X}=1 .
$$

This element need not be primitive in $H_{\mathscr{A}}$ but is often useful for computations. 
3.8. A rank 2 tube. Let $\mathscr{A}=\operatorname{rep}_{\mathbb{k}}(Q)$ where $Q$ is a valued acyclic quiver of tame type. Let $\tau$ be the Auslander-Reiten translation and consider a regular component of the Auslander-Reiten quiver which is a tube of rank 2 (that is, for every indecomposable object $M$ in that component we have $\left.\tau^{2}(M) \cong M\right)$. The smallest example is provided by the quiver

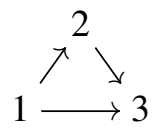

and the Auslander-Reiten component containing $S_{2}$.

Let $X$ be a simple object in our tube. Then $\tau(X)$ is also simple and both satisfy $\operatorname{End}_{\mathscr{A}} X \cong \operatorname{End}_{\mathscr{A}} \tau(X) \cong \mathbb{k}$. Furthermore,

$$
\operatorname{Ext}_{\mathscr{A}}^{1}(X, \tau(X)) \cong \operatorname{Hom}_{\mathscr{A}}(\tau(X), \tau(X)), \quad \operatorname{Ext}_{\mathscr{A}}^{1}(\tau(X), X) \cong \operatorname{Hom}_{\mathscr{A}}(X, X),
$$

and so $X, \tau(X)$ satisfy the assumptions of $\S 3.7$. Thus, we obtain a primitive element of degree $|X|+|\tau(X)|$ in the Hall algebra of our tube given by

$$
Z_{X, \tau(X)}+Z_{\tau(X), X}-(q-1)[X \oplus Y] .
$$

For the quiver shown above, with $X=S_{2}$ we have

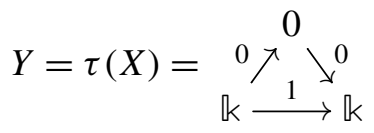

while

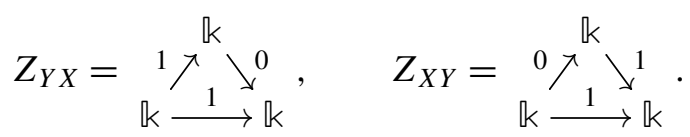

However, in $H_{\mathscr{A}}$ we have

$$
\bar{\Delta}_{\mathscr{A}}\left(Z_{Y X}+Z_{X Y}-(q-1)[X \oplus Y]\right)=(q-1)\left(\left[S_{3}\right] \otimes\left[I_{2}\right]+\left[P_{2}\right] \otimes\left[S_{1}\right]\right)
$$

where $I_{2}$ is the injective envelope of $S_{2}$ and $P_{2}$ is its projective cover. Other indecomposable objects with the same image in $\Gamma_{\mathscr{A}}$ are, up to an isomorphism,

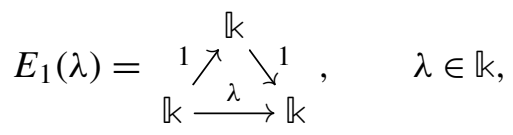

and we have

$$
\bar{\Delta}\left(E_{1}(\lambda)\right)=(q-1)\left(\left[S_{3}\right] \otimes\left[I_{2}\right]+\left[P_{2}\right] \otimes\left[S_{1}\right]\right) .
$$


This gives $q-1$ linearly independent primitive elements

$$
\mathcal{P}_{1}(\lambda)=E_{1}(\lambda)-\frac{1}{q} \sum_{\mu \in \mathbb{k}} E_{1}(\mu)
$$

and one more primitive element

$$
\left[Z_{Y X}\right]+\left[Z_{X Y}\right]-(q-1)[X \oplus Y]-\frac{1}{q} \sum_{\lambda \in \mathbb{k}} E_{1}(\lambda) .
$$

Thus, in this case $m_{\alpha_{1}+\alpha_{2}+\alpha_{3}}=2$ and $\operatorname{dim} \operatorname{Prim}\left(H_{\mathscr{A}}\right)_{\alpha_{1}+\alpha_{2}+\alpha_{3}}=q$.

In general, primitive elements in Hall algebras corresponding to nonhomogeneous tubes were computed in [Hubery 2005]. It should be noted that they are not primitive in $H_{\mathscr{A}}$ but, conjecturally, can be used to construct primitive elements in a way similar to that shown above.

3.9. Cyclic quivers with relations. Let $\mathscr{A}$ be the category of representations of the quiver

$$
1 \underset{a_{21}}{\stackrel{a_{12}}{\rightleftarrows}} 2
$$

satisfying the relation $a_{21} a_{12}=0$. The three nonsimple indecomposable objects are, up to an isomorphism,

$$
S_{12}: \mathbb{k} \underset{0}{\stackrel{1}{\rightleftarrows}} \mathbb{k}, \quad S_{21}: \mathbb{k} \underset{1}{\stackrel{0}{\rightleftarrows}} \mathbb{k}, \quad S_{212}: \mathbb{k} \underset{(01)}{\stackrel{\left(\begin{array}{l}
1 \\
0
\end{array}\right)}{\rightleftarrows}} \mathbb{k}^{2} .
$$

The object $S_{12}$ is the projective cover of $S_{1}$ while $S_{21}$ is its injective envelope. Thus,

$$
\bar{\Delta}\left(\left[S_{12}\right]\right)=(q-1)\left[S_{2}\right] \otimes\left[S_{1}\right], \quad \bar{\Delta}\left(\left[S_{21}\right]\right)=(q-1)\left[S_{1}\right] \otimes\left[S_{2}\right]
$$

and so

$$
Z=\left[S_{12}\right]+\left[S_{21}\right]-(q-1)\left[S_{1} \oplus S_{2}\right]
$$

is the unique, up to a scalar, primitive element in $\left|S_{1}\right|+\left|S_{2}\right|$. Let $E_{1}=\left[S_{1}\right]$ and $E_{2}=\left[S_{2}\right]$. Then $\operatorname{Prim}\left(H_{\mathscr{A}}\right)$ is spanned by $E_{1}, E_{2}$ and $Z$ and

$$
\left[E_{1}, Z\right]=\left[E_{2}, Z\right]=0
$$

and

$$
\left[E_{1},\left[E_{1}, E_{2}\right]_{q}\right]_{q^{-1}}=\left(1-q^{-1}\right) E_{1} Z, \quad\left[E_{2},\left[E_{2},\left[E_{2}, E_{1}\right]\right]_{q}\right]_{q^{-1}}=0
$$

is a presentation of $H_{\mathscr{A}}$.

Now let $\mathscr{A}$ be the category of representations of the same quiver satisfying the relations $a_{21} a_{12}=0=a_{12} a_{21}$. In this case, we have four indecomposable objects $S_{1}, S_{2}, S_{12}$ and $S_{21}$ which coincide with the ones listed above. Thus, $S_{i j}$ is the 
injective envelope of $S_{i}$ and the projective cover of $S_{j},\{i, j\}=\{1,2\}$. As before, we have a unique nonsimple primitive element given by the same formula (3-3). The following provides a presentation for $H_{\mathscr{A}}$ :

$$
\begin{aligned}
{\left[E_{1},\left[E_{1}, E_{2}\right]_{q}\right]_{q^{-1}} } & =\left(1-q^{-1}\right) E_{1} Z, \\
{\left[E_{2},\left[E_{2}, E_{1}\right]_{q}\right]_{q^{-1}} } & =\left(1-q^{-1}\right) E_{2} Z, \\
{\left[E_{1}, Z\right] } & =\left[E_{2}, Z\right]=0 .
\end{aligned}
$$

In both examples, we have $C_{\mathscr{A}} \subsetneq U_{\mathscr{A}}=E_{\mathscr{A}}=H_{\mathscr{A}}$ which contributes supporting evidence for Conjecture 1.13. Note also that in this case $m_{\gamma}=1$ for $\gamma=\left|S_{1}\right|+\left|S_{2}\right|$.

\section{The PBW property of Hall algebras and proof of Theorem 2.4}

4.1. Rings filtered and graded by ordered monoids. Let $(\Lambda, \triangleleft)$ be an ordered abelian monoid, as defined in $\$ 2.2$. We write $\mu \unlhd v$ if either $\mu=v$ or $\mu \neq v$ and $\mu \triangleleft v$.

Definition 4.1. We say that a unital ring $\mathcal{H}$ is $\Lambda$-filtered if $\mathcal{H}$ contains a family of abelian subgroups $\mathcal{H}^{\unlhd \lambda}, \lambda \in \Lambda^{+}$, such that for all $\lambda, \mu \in \Lambda^{+}$,

(i) $1_{\mathcal{H}} \in \mathcal{H}^{\unlhd \lambda}$ and $\lambda \unlhd \mu \Rightarrow \mathcal{H}^{\unlhd \lambda} \subset \mathcal{H}^{\unlhd \mu}$;

(ii) $\mathcal{H}=\sum_{\lambda \in \Lambda^{+}} \mathcal{H} \unlhd \lambda$;

(iii) $\mathcal{H}^{\unlhd \lambda} \cdot \mathcal{H}^{\unlhd \mu} \subset \mathcal{H}^{\unlhd(\lambda+\mu)}$.

This definition is similar to that in [Polishchuk and Positselski 2005, §4.7]; however, we do not require the ring $\mathcal{H}$ to admit a $\mathbb{Z}_{\geq 0}$-grading compatible with $\Lambda$.

Given $\lambda \in \Lambda^{+}$, let

$$
\mathcal{H}^{\triangleleft \lambda}= \begin{cases}R & \text { if } \lambda \text { is minimal, } \\ \sum_{\mu \triangleleft \lambda} \mathcal{H}^{\unlhd \mu} & \text { if } \lambda \text { is not minimal, }\end{cases}
$$

where $R=\bigcap_{\lambda \in \Lambda^{+}} \mathcal{H}^{\unlhd \lambda}$. Note that $R$ is a subring of $\mathcal{H}$ and that each $\mathcal{H}^{\unlhd \lambda}$, hence $\mathcal{H}^{\triangleleft \lambda}$, is an $R$-bimodule. We have

$$
\mathcal{H}^{\triangleleft \lambda} \cdot \mathcal{H}^{\unlhd \mu} \subset \mathcal{H}^{\triangleleft(\lambda+\mu)}, \quad \mathcal{H}^{\unlhd \lambda} \cdot \mathcal{H}^{\triangleleft \mu} \subset \mathcal{H}^{\triangleleft(\lambda+\mu)} .
$$

Define the abelian group $\operatorname{gr}_{\Lambda} \mathcal{H}$ by

$$
\operatorname{gr}_{\Lambda} \mathcal{H}=R \oplus \bigoplus_{\lambda \in \Lambda} \overline{\mathcal{H}}^{\lambda}, \quad \overline{\mathcal{H}}^{\lambda}:=\mathcal{H}^{\unlhd \lambda} / \mathcal{H}^{\triangleleft \lambda} .
$$

Lemma 4.2. The abelian group $\operatorname{gr}_{\Lambda} \mathcal{H}$ is a $\Lambda$-graded unital ring with the multiplication given by

$$
\left(x+\mathcal{H}^{\triangleleft \lambda}\right) \bullet\left(y+\mathcal{H}^{\triangleleft \mu}\right)=x \cdot y+\mathcal{H}^{\triangleleft(\mu+\nu)}, \quad \text { for } x \in \mathcal{H}^{\unlhd \lambda}, y \in \mathcal{H}^{\unlhd \mu}
$$

and $r \bullet\left(x+\mathcal{H}^{\triangleleft \lambda}\right)=r x+\mathcal{H}^{\triangleleft \lambda},\left(x+\mathcal{H}^{\triangleleft \lambda}\right) \bullet r=x r+\mathcal{H}^{\triangleleft \lambda}$ for all $x \in \mathcal{H}^{\unlhd \lambda}, r \in R$. 
Proof. By construction, the multiplication by elements of $R$ is well-defined. Using (4-1), we obtain, for all $x \in \mathcal{H}^{\unlhd \lambda}, y \in \mathcal{H}^{\unlhd \lambda}$,

$$
\left(x+\mathcal{H}^{\triangleleft \lambda}\right) \bullet\left(y+\mathcal{H}^{\triangleleft \mu}\right) \subset x \cdot y+\mathcal{H}^{\unlhd \lambda} \cdot \mathcal{H}^{\triangleleft \mu}+\mathcal{H}^{\triangleleft \lambda} \cdot \mathcal{H}^{\unlhd \mu}+\mathcal{H}^{\triangleleft \lambda} \cdot \mathcal{H}^{\triangleleft \mu} \subset x \cdot y+\mathcal{H}^{\triangleleft(\lambda+\mu)} .
$$

Thus, $\bullet$ is well-defined. The distributivity and the associativity follow from those in $\mathcal{H}$. Then the ring $\mathcal{H}$ is graded by $\Lambda$ by construction. It remains to observe that $1_{R}$ is the unity of $\operatorname{gr}_{\Lambda} \mathcal{H}$.

Corollary 4.3. For any $\Lambda$-filtered ring $\mathcal{H}$ and any collection $\lambda_{1}, \ldots, \lambda_{k} \in \Lambda$, we have

$$
\mathcal{H}^{\unlhd \lambda_{1}} \cdots \mathcal{H}^{\unlhd \lambda_{k}} /\left(\mathcal{H}^{\unlhd \lambda_{1}} \cdots \mathcal{H}^{\unlhd \lambda_{k}} \cap \mathcal{H}^{\triangleleft\left(\lambda_{1}+\cdots+\lambda_{k}\right)}\right)=\overline{\mathcal{H}}^{\lambda_{1}} \bullet \cdots \bullet \overline{\mathcal{H}}^{\lambda_{k}} .
$$

Let $\Lambda_{\text {min }}$ be the set of minimal, with respect to the partial order $\unlhd$, elements of $\Lambda^{+}$. We say that $\Lambda$ is optimal if it is generated by $\Lambda_{\min }$.

Recall that an $\mathbb{F}$-algebra $A$ is generated over its subalgebra $A_{0}$ by a subspace $A_{1} \subset A$ if $A_{1}$ is an $A_{0}$-bimodule and there exists a surjective homomorphism $T_{A_{0}}\left(A_{1}\right) \rightarrow A$ which restricts to the identity on $A_{0}+A_{1}$. Let $(\Lambda, \unlhd)$ be an optimal monoid and for any subset $\Lambda_{\circ}$ of $\Lambda_{\min }$ define

$$
\mathcal{H}_{\circ}:=\sum_{\lambda \in \Lambda_{\circ}} \mathcal{H}^{\unlhd \lambda}, \quad \overline{\mathcal{H}}_{\circ}:=\bigoplus_{\lambda \in \Lambda_{\circ}} \overline{\mathcal{H}}^{\lambda} .
$$

Lemma 4.4. Let $(\Lambda, \unlhd)$ be an optimal monoid and let $\mathcal{H}$ be a $\Lambda$-filtered ring. Let $\Lambda_{\circ} \subset \Lambda_{\text {min }}$ be a generating set for $\Lambda$ as a monoid. If $\mathcal{H}_{\circ}$ generates $\mathcal{H}$ then $\overline{\mathcal{H}}_{\circ}$ generates $\operatorname{gr}_{\Lambda} \mathcal{H}$ over $R$.

Proof. Given $x \in \mathcal{H}$, define $v(x)=\min \left\{k \geq 0: x \in \mathcal{H}_{\circ}^{k}\right\}$ where $\mathcal{H}_{\circ}^{0}=R=\overline{\mathcal{H}}_{\circ}^{0}$. Since $\operatorname{gr}_{\Lambda} \mathcal{H}$ is $\Lambda$-graded, it is sufficient to prove that for every $\bar{x} \in \overline{\mathcal{H}}^{\lambda}, \lambda \in \Lambda^{+}$we have $\bar{x} \in \overline{\mathcal{H}}_{\circ}^{\bullet k}$ for some $k$. Take $x \in \mathcal{H}^{\unlhd \lambda} \backslash \mathcal{H}^{\triangleleft \lambda}$ such that $x+\mathcal{H}^{\triangleleft \lambda}=\bar{x}$. Let $k=v(x)$. Then

$$
x \in \sum \mathcal{H}^{\unlhd \lambda_{1}} \ldots \mathcal{H}^{\unlhd \lambda_{k}},
$$

where the sum is taken over all $\left(\lambda_{1}, \ldots, \lambda_{k}\right) \in \Lambda_{\circ}^{k}$ such that $\lambda_{1}+\cdots+\lambda_{k}=\lambda$. Using Corollary 4.3 we conclude that $\bar{x} \in \sum \overline{\mathcal{H}}^{\lambda_{1}} \bullet \cdots \bullet \overline{\mathcal{H}}^{\lambda_{k}} \subset \overline{\mathcal{H}}_{\circ}^{\bullet k}$.

Proposition 4.5. Suppose that $(\Lambda, \unlhd)$ is optimal and $\triangleleft$ is inductive. Let $\Lambda_{\circ} \subset \Lambda_{\text {min }}$ be a generating set for $\Lambda$. If $\overline{\mathcal{H}}_{\circ}$ generates $\operatorname{gr}_{\Lambda} \mathcal{H}$ over $R$ then $\mathcal{H}_{\circ}$ generates $\mathcal{H}$.

Proof. Define

$$
\bar{v}(\bar{x})=\min \left\{k \geq 0: \bar{x} \in \overline{\mathcal{H}}_{\circ}^{\bullet k}\right\}
$$

for all $\bar{x} \in \operatorname{gr}_{\Lambda} \mathcal{H}$. We prove by induction on $f(\lambda), \lambda \in \Lambda^{+}$that for every $x \in \mathcal{H}^{\unlhd \lambda}$, we have $x \in \mathcal{H}_{\circ}^{k}$ for some $k \geq 0$. This is sufficient since every $x \in \mathcal{H}$ belongs to the sum of finitely many $\mathcal{H}^{\unlhd \lambda}$. 
The induction base is obvious since for $\lambda \in \Lambda_{\circ}$ we can take $k=1$. Suppose that $x \in \mathcal{H}^{\unlhd \lambda}$ for some $\lambda \in \Lambda^{+} \backslash \Lambda_{\circ}$. If $x \in \mathcal{H}^{\unlhd \mu}$ for some $\mu \triangleleft \lambda$ then we are done by the induction hypothesis. Therefore, we may assume that $x \in \mathcal{H}^{\unlhd \lambda} \backslash \mathcal{H}^{\triangleleft \lambda}$ hence $\bar{x}:=x+\mathcal{H}^{\triangleleft \lambda} \neq 0$ in $\operatorname{gr}_{\Lambda} \mathcal{H}$. Let $k=\bar{v}(\bar{x})$. Then

$$
\bar{x} \in \sum \overline{\mathcal{H}}^{\lambda_{1}} \bullet \cdots \bullet \overline{\mathcal{H}}^{\lambda_{k}},
$$

where the sum is taken over $\left(\lambda_{1}, \ldots, \lambda_{k}\right) \in \Lambda_{\circ}^{k}$ such that $\lambda_{1}+\cdots+\lambda_{k}=\lambda$. Then

$$
x \in \sum_{\substack{\left(\lambda_{1}, \ldots, \lambda_{k}\right) \in \Lambda_{\circ}^{k} \\ \lambda_{1}+\cdots+\lambda_{k}=\lambda}} \mathcal{H}^{\unlhd \lambda_{1}} \cdots \mathcal{H}^{\unlhd \lambda_{k}}+\mathcal{H}^{\triangleleft \lambda} \subset \mathcal{H}_{\circ}^{k}+\mathcal{H}^{\triangleleft \lambda} .
$$

hence $x=x^{\prime}+x^{\prime \prime}$ where $x^{\prime} \in \mathcal{H}_{\circ}^{k}, x^{\prime \prime} \in \mathcal{H}^{\triangleleft \lambda}$. Then using the definition of $\mathcal{H}^{\triangleleft \lambda}$ we can write $x^{\prime \prime}=x_{1}^{\prime \prime}+\cdots+x_{\ell}^{\prime \prime}$, where $x_{j}^{\prime \prime} \in \mathcal{H}^{\unlhd} \mu_{j}$ with $\mu_{j} \triangleleft \lambda, 1 \leq j \leq \ell$. Since $f\left(\mu_{j}\right)<f(\lambda)$, by the induction hypothesis $x_{j}^{\prime \prime} \in \mathcal{H}_{\circ}^{k_{j}^{\prime}}$ for some $k_{j}^{\prime} \geq 1$ with $1 \leq j \leq \ell$. Then $x \in \mathcal{H}_{\circ}^{\max \left(k, k_{1}^{\prime}, \ldots, k_{\ell}^{\prime}\right)}$.

Proposition 4.6 (weak PBW property). Let $(\Lambda, \unlhd)$ be an optimal monoid, let $\triangleleft$ be inductive and let $\Lambda_{\circ} \subset \Lambda_{\min }$ be a subset which generates $\Lambda$ as a monoid. Let $\mathcal{H}$ be a $\Lambda$-filtered ring. Suppose that there exists a total order $\leq$ on $\Lambda_{\circ}$ such that

$$
\operatorname{gr}_{\Lambda} \mathcal{H}=\sum_{k \geq 0} \sum_{\lambda_{1} \leq \cdots \leq \lambda_{k} \in \Lambda_{\circ}^{k}} \overline{\mathcal{H}}^{\lambda_{1}} \bullet \cdots \bullet \overline{\mathcal{H}}^{\lambda_{k}}
$$

Then

$$
\mathcal{H}=\sum_{k \geq 0} \sum_{\lambda_{1} \leq \cdots \leq \lambda_{k} \in \Lambda_{\circ}^{k}}\left(\mathcal{H}^{\unlhd \lambda_{1}}\right) \cdots\left(\mathcal{H}^{\unlhd \lambda_{k}}\right)
$$

Proof. The argument is similar to the proof of Proposition 4.5. Let

$$
\mathcal{H}^{\langle k\rangle}=\sum_{\left(\lambda_{1} \leq \cdots \leq \lambda_{k}\right) \in \Lambda_{\circ}^{k}}\left(\mathcal{H}^{\unlhd \lambda_{1}}\right) \cdots\left(\mathcal{H}^{\unlhd \lambda_{k}}\right),
$$

We prove, by induction on $f(\lambda), \lambda \in \Lambda^{+}$that for all $x \in \mathcal{H}^{\unlhd \lambda}$ there exists $k \geq 0$ such that $x \in \mathcal{H}^{\langle k\rangle}$. If $\lambda \in \Lambda_{\circ}$ then $x \in \mathcal{H}^{\langle 1\rangle}$ and we are done. Otherwise,

$$
x+\mathcal{H}^{\triangleleft \lambda} \in \sum_{\substack{\left(\lambda_{1} \leq \cdots \leq \lambda_{k}\right) \in \Lambda_{\circ}^{k} \\ \lambda_{1}+\cdots+\lambda_{k}=\lambda}} \overline{\mathcal{H}}^{\lambda_{1}} \bullet \cdots \bullet \overline{\mathcal{H}}^{\lambda_{k}},
$$

which implies that $x \in \mathcal{H}^{\langle k\rangle}+\mathcal{H}^{\triangleleft \lambda}$. Since $\mathcal{H}^{\triangleleft \lambda}=\sum_{\mu \triangleleft \lambda} \mathcal{H}^{\unlhd \mu}$, we then have $x=x^{\prime}+x_{1}^{\prime \prime}+\cdots+x_{\ell}^{\prime \prime}$ where $x^{\prime} \in \mathcal{H}^{\langle k\rangle}$ and $x_{j}^{\prime \prime} \in \mathcal{H}^{\unlhd} \mu_{j}$ for $\mu_{j} \triangleleft \lambda$ and $1 \leq j \leq \ell$. Applying the induction hypothesis to the $x_{j}^{\prime \prime}$ we conclude that $x_{j}^{\prime \prime} \in \mathcal{H}^{\left\langle k_{j}\right\rangle}$ for some $k_{j}$, $1 \leq j \leq \ell$, hence $x \in \mathcal{H}^{\left\langle\max \left(k, k_{1}, \ldots, k_{\ell}\right)\right\rangle}$.

We now consider a special case which we will later apply to Hall algebras. 
Corollary 4.7. Let $(\Lambda, \unlhd)$ be an optimal monoid and let $\triangleleft$ be an inductive order. Let $\mathcal{H}$ be a unital $\mathbb{F}$-algebra with a basis $\{[\lambda]: \lambda \in \Lambda\}$ such that $[0]=1_{\mathcal{H}}$ and

$$
[\lambda] \cdot[\mu] \in \mathbb{F}^{\times}[\lambda+\mu]+\sum_{\nu \triangleleft \lambda+\mu} \mathbb{F}[v] .
$$

for all $\lambda, \mu \in \Lambda$. Then for any subset $\Lambda_{\circ}$ of $\Lambda_{\min }$ which generates $\Lambda$ as a monoid, the set $\left[\Lambda_{\circ}\right]:=\left\{[\lambda]: \lambda \in \Lambda_{\circ}\right\}$ generates $\mathcal{H}$ as an algebra. Moreover, for any total order on $\Lambda_{\circ}$, the set $\mathbf{M}\left(\left[\Lambda_{\circ}\right]\right)$ of ordered monomials in $\left[\Lambda_{\circ}\right]$ spans $\mathcal{H}$ as an $\mathbb{F}$-vector space. Finally, if $\Lambda$ is freely generated by $\Lambda_{\circ}$ then $\mathbf{M}\left(\left[\Lambda_{\circ}\right]\right)$ is a basis of $\mathcal{H}$.

Proof. Clearly, $\mathcal{H}$ is $\Lambda$-filtered with $\mathcal{H}^{\unlhd \lambda}=\mathbb{F}\{[\mu]: \mu \unlhd \lambda\}$. In particular, $R=\mathbb{F} \cdot[0]=\mathbb{F}$. Then $\operatorname{gr}_{\Lambda} \mathcal{H}$ has a basis $\{[\overline{[\lambda]}: \lambda \in \Lambda\}$ and

$$
\overline{[\lambda]} \cdot \overline{[\mu]} \in \mathbb{F}^{\times} \overline{[\lambda+\mu]},
$$

hence $\overline{[\lambda+\mu]} \in \mathbb{F}^{\times} \overline{[\lambda]} \cdot \overline{[\mu]}$.

Let $\leq$ be any total order on $\Lambda_{\circ}$. Given $\lambda \in \Lambda$, we can write $\lambda=\lambda_{1}+\cdots+\lambda_{r}$ with $\lambda_{i} \in \Lambda_{\circ}, 1 \leq i \leq r$ and $\lambda_{1} \leq \cdots \leq \lambda_{r}$. By (4-2) we have $\overline{[\lambda]} \in \mathbb{F}^{\times} \overline{\left[\lambda_{1}\right]} \cdots \overline{\left[\lambda_{r}\right]}$. Taking into account that $\mathcal{H} \unlhd \lambda=\mathbb{F}+\mathbb{F}[\lambda]$ for $\lambda \in \Lambda_{\circ}$, we see that all assumptions of Proposition 4.6 are satisfied.

4.2. Proof of Theorem 2.2. The key ingredient of our argument is the following result.

Proposition 4.8. Let $\mathscr{A}$ be a Hom-finite exact category. Then for any short exact sequence

$$
M^{-} \stackrel{f_{-}}{\longrightarrow} M \stackrel{f_{+}}{\longrightarrow} M^{+}
$$

we have

$$
e([M]) \leq e\left(\left[M^{+} \oplus M^{-}\right]\right),
$$

where $e([X]):=\# \operatorname{End}_{\mathscr{A}} X$ for $[X] \in$ Iso $\mathscr{A}$. Moreover, if (4-4) is an equality then (4-3) splits.

Proof. We need to prove that the following inequalities hold for every $N$ in $\mathscr{A}$ :

$$
\begin{aligned}
& \# \operatorname{Hom}_{\mathscr{A}}(N, M) \leq \# \operatorname{Hom}\left(N, M^{+} \oplus M^{-}\right), \\
& \# \operatorname{Hom}_{\mathscr{A}}(M, N) \leq \# \operatorname{Hom}\left(M^{+} \oplus M^{-}, N\right) .
\end{aligned}
$$

To prove the first inequality, recall (see, e.g., [Buchsbaum 1959; Yoneda 1954]) that for every $N$ in $\mathscr{A},(4-3)$ induces a long exact sequence of finite abelian groups

$0 \rightarrow \operatorname{Hom}_{\mathscr{A}}\left(N, M^{-}\right) \rightarrow \operatorname{Hom}_{\mathscr{A}}(N, M) \rightarrow \operatorname{Hom}_{\mathscr{A}}\left(N, M^{+}\right) \stackrel{\delta_{*}}{\rightarrow}$

$$
\operatorname{Ext}_{\mathscr{A}}^{1}\left(N, M^{-}\right) \rightarrow \operatorname{Ext}_{\mathscr{A}}^{1}(N, M) \rightarrow \operatorname{Ext}_{\mathscr{A}}^{1}\left(N, M^{+}\right) \rightarrow \cdots .
$$


Truncating this sequence yields an exact sequence

(4-6) $0 \rightarrow \operatorname{Hom}_{\mathscr{A}}\left(N, M^{-}\right) \rightarrow \operatorname{Hom}_{\mathscr{A}}(N, M) \rightarrow \operatorname{Hom}_{\mathscr{A}}\left(N, M^{+}\right) \stackrel{\delta_{*}^{*}}{\rightarrow} \operatorname{Im} \delta_{*} \rightarrow 0$.

Then, computing the multiplicative Euler characteristic of (4-6), we obtain

$$
\begin{aligned}
\# \operatorname{Hom}_{\mathscr{A}}\left(N, M^{-}\right) \cdot \# \operatorname{Hom}_{\mathscr{A}}\left(N, M^{+}\right) & =\# \operatorname{Hom}_{\mathscr{A}}(N, M) \cdot \# \operatorname{Im} \delta_{*} \\
& \geq \# \operatorname{Hom}_{\mathscr{A}}(N, M),
\end{aligned}
$$

which immediately yields the first inequality in (4-5).

To prove the second inequality, recall that for all $N$ in $\mathscr{A},(4-3)$ induces a long exact sequence of abelian finite groups

$$
\begin{aligned}
0 \rightarrow \operatorname{Hom}_{\mathscr{A}}\left(M^{+}, N\right) \rightarrow & \operatorname{Hom}_{\mathscr{A}}(M, N) \rightarrow \operatorname{Hom}_{\mathscr{A}}\left(M^{-}, N\right) \stackrel{\delta^{*}}{\rightarrow} \\
& \operatorname{Ext}_{\mathscr{A}}^{1}\left(M^{+}, N\right) \rightarrow \operatorname{Ext}_{\mathscr{A}}^{1}(M, N) \rightarrow \operatorname{Ext}_{\mathscr{A}}^{1}\left(M^{-}, N\right) \rightarrow \cdots .
\end{aligned}
$$

Similarly, truncating this sequence yields

$$
0 \rightarrow \operatorname{Hom}_{\mathscr{A}}\left(M^{+}, N\right) \rightarrow \operatorname{Hom}_{\mathscr{A}}(M, N) \rightarrow \operatorname{Hom}_{\mathscr{A}}\left(M^{-}, N\right) \stackrel{\delta^{*}}{\rightarrow} \operatorname{Im} \delta^{*} \rightarrow 0,
$$

and the argument identical to the above gives

$$
\# \operatorname{Hom}_{\mathscr{A}}(M, N) \leq \# \operatorname{Hom}_{\mathscr{A}}\left(M^{+}, N\right) \cdot \# \operatorname{Hom}_{\mathscr{A}}\left(M^{-}, N\right)
$$

which is equivalent to the second inequality in (4-5).

Combining the first inequality in (4-5) with $N=M^{+} \oplus M^{-}$and the second inequality in (4-5) with $N=M$ we obtain

$$
\begin{aligned}
e([M])=\# \operatorname{End}_{\mathscr{A}} M & \leq \# \operatorname{Hom}_{\mathscr{A}}\left(M^{+} \oplus M^{-}, M\right) \\
& \leq \# \operatorname{End}_{\mathscr{A}} M^{+} \oplus M^{-}=e\left(\left[M^{+} \oplus M^{-}\right]\right) .
\end{aligned}
$$

To prove the last assertion, it suffices to show, in view of the above chain of inequalities, that $\# \operatorname{Hom}_{\mathscr{A}}\left(M^{+} \oplus M^{-}, M\right)=\# \operatorname{End}_{\mathscr{A}} M^{+} \oplus M^{-}$implies that (4-3) splits. Indeed, using the additivity of $\operatorname{Hom}_{\mathscr{A}}$ in the first argument we rewrite the latter equality as

$$
\begin{aligned}
\# \operatorname{Hom}_{\mathscr{A}}\left(M^{+}, M\right) \cdot \# \operatorname{Hom}_{\mathscr{A}}\left(M^{-}, M\right) \\
\quad=\# \operatorname{Hom}_{\mathscr{A}}\left(M^{+}, M^{+} \oplus M^{-}\right) \cdot \# \operatorname{Hom}_{\mathscr{A}}\left(M^{-}, M^{+} \oplus M^{-}\right) .
\end{aligned}
$$

This and (4-5) taken with $N=M^{-}$imply

$$
\# \operatorname{Hom}_{\mathscr{A}}\left(M^{+}, M\right) \geq \# \operatorname{Hom}_{\mathscr{A}}\left(M^{+}, M^{+} \oplus M^{-}\right),
$$

which, together with (4-5) with $N=M^{+}$, yield

$$
\# \operatorname{Hom}_{\mathscr{A}}\left(M^{+}, M\right)=\# \operatorname{Hom}_{\mathscr{A}}\left(M^{+}, M^{+} \oplus M^{-}\right) .
$$


The last equality and (4-7) taken with $N=M^{+}$imply that $E_{0}=\operatorname{Im} \delta_{*}=0$, hence the natural map $\operatorname{Hom}_{\mathscr{A}}\left(M^{+}, M\right) \rightarrow \operatorname{End}_{\mathscr{A}} M^{+}$is surjective. Therefore, there exists $g \in \operatorname{Hom}_{\mathscr{A}}\left(M^{+}, M\right)$ such that $f_{+} \circ g=1_{M^{+}}$, hence (4-3) splits.

Recall that $\triangleleft$ is the preorder defined as the transitive closure of the relation

$$
[M] \triangleleft\left[M^{-} \oplus M^{+}\right] \Longleftrightarrow \exists \text { a nonsplit short exact sequence } M^{-} \longmapsto M \rightarrow M^{+}
$$

(cf. §2.2). By Proposition 4.8, $\triangleleft$ is an inductive preorder with the function mapping $[X]$ to $e([X])$, hence is an inductive partial order.

It remains to prove that the order $\triangleleft$ is compatible with the addition in Iso $\mathscr{A}$. Indeed, note that for any $X$ in $\mathscr{A}$, the short exact sequence (4-3) yields a short exact sequence

$$
M^{-} \oplus X \stackrel{\left(\begin{array}{cc}
f_{-} & 0 \\
0 & 1_{X}
\end{array}\right)}{\longrightarrow} M \oplus X \stackrel{\left(f_{+}, 0\right)}{\longrightarrow} M^{+},
$$

hence $[M \oplus X] \unlhd\left[M^{-} \oplus M^{+} \oplus X\right]$. If $[M] \triangleleft\left[M^{-} \oplus M^{+}\right]$, that is, (4-3) is nonsplit, then clearly (4-8) is also nonsplit, so $[M \oplus X] \triangleleft\left[M^{-} \oplus M^{+} \oplus X\right]$. Taking transitive closure implies that $[M \oplus X] \triangleleft[N \oplus X]$ for all $[M],[N] \in$ Iso $\mathscr{A}$ such that $[M] \triangleleft[N]$ and for all $[X] \in$ Iso $\mathscr{A}$. This completes the proof of Theorem 2.2.

4.3. Proof of Theorem 2.4. We are now going to apply the machinery developed in $\S 4.1$. We begin by proving that (Iso $\mathscr{A}, \triangleleft$ ) is optimal.

Lemma 4.9. Let $\mathscr{A}$ be an exact Hom-finite category. Then every object $X$ in $\mathscr{A}$ is a finite direct sum of indecomposable objects and the number of indecomposable summands of $X$ is bounded above by \# End $\mathscr{A} X$.

Proof. Let $X$ be a nonzero object in $\mathscr{A}$. Write $X=X_{1} \oplus \cdots \oplus X_{s}$ for some $s>0$, where all the $X_{i}$ are nonzero. Then \# $\operatorname{End}_{\mathscr{A}} X \geq \sum_{j=1}^{s} \# \operatorname{End}_{\mathscr{A}} X_{i} \geq s$. Let $k$ be the maximal positive integer $s$ such that $X$ can be written as a direct sum of $s$ nonzero objects. The maximality of $k$ immediately implies that each summand is indecomposable.

Remark 4.10. It should be noted that the Krull-Schmidt theorem does not have to hold in this generality. For example, the full subcategory of the category of $\mathbb{k}$-representations of the quiver $1 \rightarrow 0 \leftarrow 2$, with the dimension vector satisfying $\operatorname{dim}_{\mathbb{k}} V_{1}=\operatorname{dim}_{\mathbb{k}} V_{2}$, is not Krull-Schmidt.

Corollary 4.11. The monoid Iso $\mathscr{A}$ is generated by Ind $\mathscr{A}$ and is optimal with respect to $\unlhd$.

Proof. The first assertion is immediate from the lemma. To prove the second, observe that if $[N]$ is not minimal, then $[M] \triangleleft[N]$ for some $[M] \in$ Iso $\mathscr{A}$ and so $N$ is decomposable. Thus, every $[X] \in$ Ind $\mathscr{A}$ is minimal with respect to the partial order $\unlhd$, hence Iso $\mathscr{A}$ is generated by its minimal elements. 
Proof of Theorem 2.4. Since (Iso $\mathscr{A}, \triangleleft$ ) is optimal and $\triangleleft$ is inductive, the algebra $H_{\mathscr{A}}$ satisfies the assumptions of Corollary 4.7 with $\Lambda=$ Iso $\mathscr{A}$ and $\Lambda_{\circ}=$ Ind $\mathscr{A}$. Therefore, for any total order on Ind $\mathscr{A}$, ordered monomials on Ind $\mathscr{A}$ span $H_{\mathscr{A}}$. Finally, if $\mathscr{A}$ is Krull-Schmidt, Iso $\mathscr{A}$ is freely generated by Ind $\mathscr{A}$, hence ordered monomials on Ind $\mathscr{A}$ form a basis of $H_{\mathscr{A}}$.

\section{The Grothendieck monoid of a profinitary category}

5.1. Almost simple objects. We will repeatedly need the following obvious description of the defining relation of the Grothendieck monoid.

Lemma 5.1. Suppose that $[X] \neq[Y] \in(\text { Iso } \mathscr{A})^{+}$and $|X|=|Y|$. Then there exist $\left[X_{i}\right] \in(\text { Iso } \mathscr{A})^{+}, 0 \leq i \leq r$ and $\left[A_{i}\right],\left[B_{i}\right] \in(\text { Iso } \mathscr{A})^{+}, 1 \leq i \leq r$ such that $\left[X_{0}\right]=[X]$, $\left[X_{r}\right]=[Y]$ and $\left[X_{i-1}\right],\left[X_{i}\right] \in \underline{\operatorname{Ext}}_{\mathscr{A}}^{1}\left(A_{i}, B_{i}\right), 1 \leq i \leq r$.

Definition 5.2. We say that an object $X \neq 0$ in an exact category $\mathscr{A}$ is almost simple if there is no nontrivial short exact sequence $Y \longmapsto X \rightarrow Z$ (or, equivalently, $\left.[X] \in \underline{\operatorname{Ext}}_{\mathscr{A}}^{1}(A, B) \Longrightarrow\{[A],[B]\}=\{[X],[0]\}\right)$ and simple if it has no proper nonzero subobjects.

Clearly, in an abelian category these notions coincide. Note that an almost simple object is always indecomposable. Let $S_{\mathscr{A}} \subset$ Iso $\mathscr{A}$ be the set of isomorphism classes of almost simple objects. The definition (2-3) of comultiplication $\Delta$ implies that

$$
F_{X}^{A B}= \begin{cases}1 & \text { if }\{[A],[B]\}=\{[X],[0]\} \\ 0 & \text { otherwise }\end{cases}
$$

hence

$$
S_{\mathscr{A}} \subset \operatorname{Prim}\left(H_{\mathscr{A}}\right) .
$$

Let $\Gamma$ be an abelian monoid. Observe that the elements of $\Gamma^{+} \backslash\left(\Gamma^{+}+\Gamma^{+}\right)$are precisely the minimal elements of $\Gamma^{+}$in the preorder $\preceq$ (cf. §2.4).

Lemma 5.3. Let $\mathscr{A}$ be an exact category. Then the restriction of the canonical homomorphism of monoids $\phi_{\mathscr{A}}:$ Iso $\mathscr{A} \rightarrow \Gamma_{\mathscr{A}}$ to $S_{\mathscr{A}}$ is a bijection

$$
S_{\mathscr{A}} \rightarrow \Gamma_{\mathscr{A}}^{+} \backslash\left(\Gamma_{\mathscr{A}}^{+}+\Gamma_{\mathscr{A}}^{+}\right) \text {. }
$$

In particular, if $\mathscr{A}$ is Hom-finite, then $\left(H_{\mathscr{A}}\right)_{\gamma}$ equals $\operatorname{Prim}\left(H_{\mathscr{A}}\right)_{\gamma}$ and is onedimensional for all $\gamma \in \Gamma_{\mathscr{A}}^{+} \backslash\left(\Gamma_{\mathscr{A}}^{+}+\Gamma_{\mathscr{A}}^{+}\right)$.

Proof. Lemma 5.1 implies that for $[X] \in S_{\mathscr{A}}$, we have $|X|=|Y|$ if and only if $[X]=[Y]$. This shows that the restriction of $\phi_{\mathscr{A}}$ to $S_{\mathscr{A}}$ is injective. Furthermore, if $|X|=|Y|+|Z|=|Y \oplus Z|$ for some nonzero $[Y],[Z]$ then $[X]=[Y \oplus Z]$, which is a contradiction since $X$ is indecomposable. Thus, $\operatorname{Im} \phi_{\mathscr{A}} \subset \Gamma_{\mathscr{A}}^{+} \backslash\left(\Gamma_{\mathscr{A}}^{+}+\Gamma_{\mathscr{A}}^{+}\right)$and so the map in (5-2) is well-defined. Finally, if $[X] \notin S_{\mathscr{A}}$, then $[X] \in \underline{\operatorname{Ext}}_{\mathscr{A}}^{1}(A, B)$ 
with $|A|,|B| \in \Gamma_{\mathscr{A}}^{+}$, hence $|X|=|A|+|B| \in \Gamma_{\mathscr{A}}^{+}+\Gamma_{\mathscr{A}}^{+}$. Thus, the preimage of $\Gamma_{\mathscr{A}}^{+} \backslash\left(\Gamma_{\mathscr{A}}^{+}+\Gamma_{\mathscr{A}}^{+}\right)$is contained in $S_{\mathscr{A}}$ hence $\left.\phi_{\mathscr{A}}\right|_{S_{\mathscr{A}}}$ is surjective.

In particular, $\operatorname{dim}_{\mathbb{Q}}\left(H_{\mathscr{A}}\right)_{\gamma}=\#$ Iso $\mathscr{A}_{\gamma}=1$ for all $\gamma \in \Gamma_{\mathscr{A}}^{+} \backslash\left(\Gamma_{\mathscr{A}}^{+}+\Gamma_{\mathscr{A}}^{+}\right)$. The equality $\left(H_{\mathscr{A}}\right)_{\gamma}=\operatorname{Prim}\left(H_{\mathscr{A}}\right)_{\gamma}$ now follows from (5-1).

Remark 5.4. Note that we can have $\operatorname{dim}_{\mathbb{Q}}\left(H_{\mathscr{A}}\right)_{\gamma}=1$ even for $\gamma \in \Gamma^{+}+\Gamma^{+}$. For example, if $S, S^{\prime}$ are simple objects with $\operatorname{Ext}_{\mathscr{A}}^{1}\left(S, S^{\prime}\right)=\operatorname{Ext}_{\mathscr{A}}^{1}\left(S^{\prime}, S\right)=0$, then $\left(H_{\mathscr{A}}\right)_{|S|+\left|S^{\prime}\right|}=\mathbb{Q}\left[S \oplus S^{\prime}\right]=\mathbb{Q}[S]\left[S^{\prime}\right]$. However, in that case Prim $\left(H_{\mathscr{A}}\right)_{\gamma}=0$.

\subsection{Proof of Proposition 2.12. Let}

$$
\Gamma_{\mathscr{A}}^{f}=\left\{\gamma \in \Gamma_{\mathscr{A}}: \# \text { Iso } \mathscr{A}_{\gamma}<\infty\right\} .
$$

Thus, $\mathscr{A}$ is profinitary if $\Gamma_{\mathscr{A}}=\Gamma_{\mathscr{A}}^{f}$. Note, however, that $\Gamma_{\mathscr{A}}^{f}$ need not be a submonoid of $\Gamma_{\mathscr{A}}$. Since \# Iso $\mathscr{A}_{\gamma}=1$ for $\gamma \in \Gamma_{\mathscr{A}}^{+}$minimal, all minimal elements of $\Gamma_{\mathscr{A}}$ are

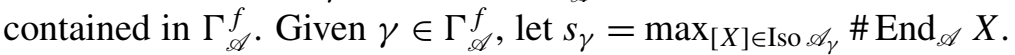

Proposition 2.12 is a special case of the following proposition.

Proposition 5.5. Let $\mathscr{A}$ be a Hom-finite exact category. Then the restriction of the preorder $\preceq$ to $\Gamma_{\mathscr{A}}^{f}$ is a partial order. Moreover, $\Gamma_{\mathscr{A}}^{f}$ is contained in the submonoid of $\Gamma_{\mathscr{A}}$ generated by its minimal elements.

Proof. We need the following lemma.

Lemma 5.6. Let $\gamma \in \Gamma_{\mathscr{A}}^{f} \backslash\{0\}$. Then $\gamma$ can be written as a sum of finitely many minimal elements of $\Gamma_{\mathscr{A}}^{+}$and the number of summands in any such presentation is bounded by $s_{\gamma}$.

Proof. The proof is almost identical to that of Lemma 4.9. Write $\gamma=\gamma_{1}+\cdots+\gamma_{s}$ for some $\gamma_{i} \in \Gamma_{\mathscr{A}}^{+}$. Take $X_{i} \in \mathscr{A}$ with $\left|X_{i}\right|=\gamma_{i}$ and let $X=X_{1} \oplus \cdots \oplus X_{s}$. Then $s$ cannot exceed the maximal possible number of indecomposable summands of $X$ which, by Lemma 5.3, is bounded above by \# $\operatorname{End}_{\mathscr{A}} X \leq s_{\gamma}$. Let $k$ be the maximal integer $s$ such that $\gamma$ can be written as a sum of $s$ elements of $\Gamma_{\mathscr{A}}^{+}$. Then the maximality of $k$ implies that each summand is minimal.

It follows from Lemma 5.6 that for $\alpha \in \Gamma_{\mathscr{A}}^{f}, \alpha=\alpha+\beta$ implies that $\beta=0$. Then $\alpha+\beta+\gamma=\alpha$ implies that $\beta+\gamma=0$, hence $\beta=\gamma=0$ since 0 is the only invertible element of $\Gamma_{\mathscr{A}}$. The first assertion of the proposition now follows from Lemma 2.10, while the second is immediate from Lemma 5.6.

5.3. Proofs of Theorems 1.4, 2.14 and Corollary 1.5. We begin with Theorem 1.4. 
Proof of Theorem 1.4. Since $[A \oplus B] \in \underline{\operatorname{Ext}}_{\mathscr{A}}^{1}(A, B)$, Definition 2.11 implies that a profinitary category $\mathscr{A}$ is cofinitary if and only if for any $\gamma \in \Gamma_{\mathscr{A}}$ the set

$$
\begin{aligned}
\mathcal{E}_{\gamma}:=\{([A],[B]) & \in \operatorname{Iso} \mathscr{A} \times \text { Iso } \mathscr{A}:|A|+|B|=\gamma\} \\
& =\bigcup_{[X] \in \operatorname{Iso} \mathscr{A}:|X|=\gamma}\left\{([A],[B]) \in \operatorname{Iso} \mathscr{A} \times \operatorname{Iso} \mathscr{A}:[X] \in \underline{\operatorname{Ext}}_{\mathscr{A}}^{1}(A, B)\right\}
\end{aligned}
$$

is finite. On the other hand,

$$
\mathcal{E}_{\gamma}=\bigcup_{\alpha, \beta \in \Gamma \mathscr{A}: \alpha+\beta=\gamma} \text { Iso } \mathscr{A}_{\alpha} \times \text { Iso } \mathscr{A}_{\beta} .
$$

Therefore, $\mathcal{E}_{\gamma}$ is finite if and only if $\left\{(\alpha, \beta) \in \Gamma_{\mathscr{A}} \times \Gamma_{\mathscr{A}}: \alpha+\beta=\gamma\right\}$ is finite.

Now we proceed to prove Theorem 2.14. Given an object $X \in \mathscr{A}$, an admissible flag on $X$ is a sequence of objects $X_{0}=X, X_{1}, \ldots, X_{s}=0$ together with short exact sequences $X_{i} \longmapsto X_{i-1} \longrightarrow Y_{i}, 1 \leq i \leq s$. An admissible flag is said to be a composition series if the $Y_{i}$ are almost simple for all $1 \leq i \leq s$.

Proposition 5.7. Let $\mathscr{A}$ be a profinitary exact category. Suppose that $\gamma \in \Gamma_{\mathscr{A}} \backslash\{0\}$. Then $X \in \mathscr{A}$ with $|X|=\gamma$ admits a composition series. Moreover, the length of any composition series of $X$ is bounded above by $s_{\gamma}$.

Proof. We use induction on the partially ordered set $\left(\Gamma_{\mathscr{A}}, \preceq\right.$ ) (see Proposition 5.5). If $\gamma \in \Gamma_{\mathscr{A}}$ is minimal then $X$ with $|X|=\gamma$ is almost simple by (5-2), and hence admits a composition series. Suppose the assertion is established for all $\gamma^{\prime} \prec \gamma$ and $\gamma$ is not minimal. Then $X$ with $|X|=\gamma$ is not almost simple, hence there exists a short exact sequence $X^{\prime \prime} \longmapsto X \stackrel{h}{\longrightarrow} X^{\prime}$ with $\left|X^{\prime}\right|,\left|X^{\prime \prime}\right| \prec|X|$. By the induction hypothesis there exists a short exact sequence $Y^{\prime \prime} \longleftrightarrow X^{\prime} \stackrel{g}{\longrightarrow} Y$ with $Y$ almost simple. Let $Y_{1}=Y$. Then we have a short exact sequence

$$
X_{1} \longmapsto X \stackrel{g h}{\longrightarrow} Y_{1}
$$

where $\left|X_{1}\right| \prec|X|$. Therefore, $X_{1}$ admits a composition series by the induction hypothesis, which establishes the first assertion of the lemma. The second assertion is immediate from Lemma 5.6 since $|X|=\left|Y_{1}\right|+\cdots+\left|Y_{S}\right|$.

Proof of Theorem 2.14. If $\mathscr{A}$ is profinitary and abelian, then the composition series from Proposition 5.7 is a composition series in the usual sense since all almost simple objects are simple. Theorem 2.14 is now immediate.

Proof of Corollary 1.5. Since a full exact subcategory of a cofinitary exact category is also cofinitary, to prove (a), it suffices to consider the case when $\mathscr{A}$ is a profinitary abelian category. Note that the uniqueness of composition factors in an abelian category with the finite length property (see, e.g., [Joyce 2006, Theorem 2.7]) implies that $\Gamma_{\mathscr{A}}$ is freely generated by its minimal elements. It remains to apply 
Theorem 1.4. To prove (b), note that by Lemma 5.6, $\Gamma_{\mathscr{A}}$ is finitely generated if and only if it contains finitely many minimal elements $\gamma_{1}, \ldots, \gamma_{n}$. Again by Lemma 5.6, the number of decompositions of $\gamma \in \Gamma_{\mathscr{A}}$ as $\gamma=\sum_{i=1}^{n} c_{i} \gamma_{i}, c_{i} \in \mathbb{Z}_{\geq 0}$ is bounded above by $\left(\begin{array}{c}s_{\gamma}+n \\ n\end{array}\right)$, which is the number of $n$-tuples $\left(c_{1}, \ldots, c_{n}\right) \in \mathbb{Z}_{\geq 0}^{n}$ with $\sum_{i=1}^{n} c_{i} \leq s_{\gamma}$. The assertion is now immediate from Theorem 1.4.

\section{Coalgebras in tensor categories and proof of the main theorem}

6.1. Quasiprimitive elements and coideals. Let $\mathbb{F}$ be a field of characteristic zero. Let $H_{0}$ be a bialgebra over $\mathbb{F}$ and let $\mathscr{C}$ be the category of left $H_{0}$-comodules. Given $V \in \mathscr{C}$, we denote the left coaction of $H_{0}$ by $\delta_{V}: V \rightarrow H_{0} \otimes V$ and, using Sweedler-like notation, write

$$
\delta_{V}(v)=v^{(-1)} \otimes v^{(0)}, \quad v \in V .
$$

The category $\mathscr{C}$ is an $\mathbb{F}$-linear tensor category with the unit object $\mathbb{F}$, the tensor product $A \otimes B=A \otimes_{\mathbb{F}} B$ of objects $A, B \in \mathscr{C}$ acquiring a left $H_{0}$-comodule structure via

$$
\delta_{A \otimes B}(a \otimes b)=a^{(-1)} b^{(-1)} \otimes a^{(0)} \otimes b^{(0)},
$$

for all $a \in A, b \in B$.

By definition, a coalgebra in $\mathscr{C}$ is an object $C \in \mathscr{C}$ together with morphisms $\Delta \in \operatorname{Hom}_{\mathscr{C}}(C, C \otimes C)$ and $\varepsilon \in \operatorname{Hom}_{\mathscr{C}}(C, \mathbb{F})$ satisfying the usual axioms. For any coalgebra $C$ in $\mathscr{C}$, denote by $C_{0}=\operatorname{Corad}_{\mathscr{C}}(C)$ the sum of all simple finite dimensional subcoalgebras of $C$ in $\mathscr{C}$ and refer to it as the coradical of $C$ in $\mathscr{C}$. Clearly, $C_{0}$ is a subcoalgebra of $C$ in $\mathscr{C}$. Denote also

$$
C_{1}=\operatorname{QPrim}_{\mathscr{C}}(C)=\Delta^{-1}\left(C \otimes C_{0}+C_{0} \otimes C\right)
$$

and refer to it as the quasiprimitive set of $C$. Then $C_{1}$ is a $\mathscr{C}$-subobject of $C$. It is well-known (see [Sweedler 1969, Corollary 9.1.7]) that

$$
\Delta\left(C_{1}\right) \subset C_{1} \otimes C_{0}+C_{0} \otimes C_{1} .
$$

In particular, if $C_{0}=\mathbb{F}$ then $\operatorname{QPrim}_{\mathscr{C}}(C)=\mathbb{F} \oplus \operatorname{Prim}(C)$. More generally, we have the following lemma which extends a well-known result (cf. [Montgomery 1993, Theorem 5.2.2; Sweedler 1969, §9.1]).

Lemma 6.1. Any coalgebra $C$ in $\mathscr{C}$ admits an increasing coradical filtration by subcoalgebras $C_{k} \subset C$ in $\mathscr{C}, k \geq 0$, defined by $C_{0}=\operatorname{Corad}_{\mathscr{C}}(C), C_{1}=\operatorname{QPrim}_{\mathscr{C}}(C)$ and

$$
C_{k}=\Delta^{-1}\left(C \otimes C_{k-1}+C_{0} \otimes C\right)
$$

for $k>1$. Moreover, $\Delta\left(C_{k}\right)=\sum_{i=0}^{k} C_{i} \otimes C_{k-i}$. 
A coideal in $C$ is a $\mathscr{C}$-subobject $I$ of $C$ satisfying

$$
\Delta(I) \subset C \otimes I+I \otimes C .
$$

Proposition 6.2. Let $C$ be a coalgebra in $\mathscr{C}$. Then for any nonzero coideal I in $\mathscr{C}$ one has

$$
I \cap \operatorname{QPrim}_{\mathscr{C}}(C) \neq\{0\} .
$$

Proof. For each $k \geq 0$ denote $I_{k}:=I \cap C_{k}$. If $I_{0} \neq\{0\}$, then we are done since $I_{0} \subset C_{0} \subset C_{1}$. Assume that $I_{0}=0$. Since $C_{0} \subset C_{1} \subset \cdots$ is a filtration, there exists a unique $k \geq 1$ such that $I_{k-1}=0$ and $I_{k} \neq 0$. Then

$$
\Delta\left(I_{k}\right) \subset C_{0} \otimes I_{k}+I_{k} \otimes C_{0} .
$$

Since $C_{1}$ is the maximal subobject $V$ of $C$ with the property $\Delta(V) \subset C_{0} \otimes V+V \otimes C_{0}$, it follows that $I_{k} \subset C_{1}$ and so $k=1$. Thus, $I_{1}=I \cap C_{1}=I \cap \operatorname{QPrim}_{\mathscr{C}}(C) \neq\{0\}$.

6.2. Invariant pairing. Given two objects $A, B$ in $\mathscr{C}$, a pairing $\langle\cdot, \cdot\rangle: A \otimes B \rightarrow \mathbb{F}$ is called $H_{0}$-invariant if

$$
a^{(-1)}\left\langle a^{(0)}, b\right\rangle=b^{(-1)}\left\langle a, b^{(0)}\right\rangle
$$

for all $a \in A, b \in B$.

The following example plays a fundamental role in the sequel.

Example 6.3. Let $\Gamma$ be an abelian monoid. Its monoidal algebra $H_{0}=\mathbb{F} \Gamma$ has a natural coalgebra structure, with the elements of $\Gamma$ being group-like. Then a left $H_{0}$-comodule $V$ is in fact a $\Gamma$-graded vector space, since $V=\bigoplus_{\gamma \in \Gamma} V_{\gamma}$ where $V_{\gamma}=\left\{v \in V: \delta_{V}(v)=\gamma \otimes v\right\}$. It is easy to see that a pairing $\langle\cdot, \cdot\rangle: A \otimes B \rightarrow \mathbb{F}$ is $H_{0}$-invariant if and only if $\left\langle A_{\gamma}, B_{\gamma^{\prime}}\right\rangle=0, \gamma \neq \gamma^{\prime} \in \Gamma$.

Lemma 6.4. Let $\langle\cdot, \cdot\rangle: A \otimes B \rightarrow \mathbb{F}$ be an $H_{0}$-invariant pairing between objects $A$ and $B$ of $\mathscr{C}$. Then for any subobject $A_{0}$ of $A$ in $\mathscr{C}$, its right orthogonal complement

$$
A_{0}{ }^{\perp}=\left\{b \in B:\left\langle A_{0}, b\right\rangle=0\right\}
$$

is a subobject of $B$ in $\mathscr{C}$. Likewise, for any subobject $B_{0}$ of $B$ in $\mathscr{C}$, its left orthogonal complement

$$
{ }^{\perp} B_{0}=\left\{a \in A:\left\langle a, B_{0}\right\rangle=0\right\}
$$

is a subobject of $A$ in $\mathscr{C}$.

Proof. We prove the first assertion only, the argument for the second one being similar. Given $b \in A_{0}{ }^{\perp}$, write $\delta_{B}(b)=\sum_{i} h_{i} \otimes b_{i}$ where the $h_{i} \in H_{0}$ are linearly independent and $b_{i} \in B$. Since the pairing is invariant, we have for all $a \in A_{0}$

$$
\sum_{i} h_{i}\left\langle a, b_{i}\right\rangle=a^{(-1)}\left\langle a^{(0)}, b\right\rangle=0
$$


since $\delta_{A}(a)=a^{(-1)} \otimes a^{(0)} \in H_{0} \otimes A_{0}$. Therefore, $\left\langle a, b_{i}\right\rangle=0$ for all $i$, hence $b_{i} \in A_{0}{ }^{\perp}$ and so $\delta_{B}(b) \in H_{0} \otimes A_{0}{ }^{\perp}$.

We now prove that an $H_{0}$-invariant pairing between nonisomorphic simple objects in $\mathscr{C}$ must be identically zero. For that purpose, it will be convenient to introduce the dual picture. Let $H_{0}^{*}=\operatorname{Hom}_{\mathbb{F}}\left(H_{0}, \mathbb{F}\right)$. Then $H_{0}^{*}$ is an associative $\mathbb{F}$-algebra via $f \cdot g=(f \otimes g) \circ \Delta_{H_{0}}$ for all $f, g \in H_{0}^{*}$, where $\Delta_{H_{0}}: H_{0} \rightarrow H_{0} \otimes H_{0}$ is the comultiplication on $H_{0}$ (hereafter we identify $\mathbb{E} \otimes_{\mathbb{F}} V$ with $V$ via the canonical isomorphism). Then a left $H_{0}$-comodule $V$ is naturally a left $H_{0}^{*}$-module via $f \triangleright v=(f \otimes 1) \delta_{V}(v)$, for all $f \in H_{0}^{*}$ and $v \in V$. This yields a fully faithful functor from the category $\mathscr{C}$ to the category of left $H_{0}^{*}$-modules. In particular, $V \cong V^{\prime}$ in $\mathscr{C}$ if and only if they are isomorphic as $H_{0}^{*}$-modules. If $\langle\cdot, \cdot\rangle: A \otimes B \rightarrow \mathbb{F}$ is an $H_{0}$-invariant pairing, then for all $a \in A, b \in B$ and $f \in H_{0}^{*}$ we have

$$
\begin{aligned}
\langle f \triangleright a, b\rangle & =f\left(a^{(-1)}\right)\left\langle a^{(0)}, b\right\rangle \\
& =f\left(b^{(-1)}\right)\left\langle a, b^{(0)}\right\rangle=\langle a, f \triangleright b\rangle .
\end{aligned}
$$

Finally, note that $V$ is a simple $H_{0}$-comodule if and only if it is simple as a left $H_{0}^{*}$-module.

Proposition 6.5. Let $A$ and $B$ be simple objects in $\mathscr{C}$. Let $\langle\cdot, \cdot\rangle: A \otimes B \rightarrow \mathbb{F}$ be a nonzero $H_{0}$-invariant pairing. Then $A \cong B$ in $\mathscr{C}$.

Proof. Given $a \in A$, let $J_{a}=\operatorname{Ann}_{H_{0}^{*}} a=\left\{f \in H_{0}^{*}: f \triangleright a=0\right\}$. We need the following technical result.

Lemma 6.6. Let $A, B$ be objects in $\mathscr{C}$ and let $\langle\cdot, \cdot\rangle: A \otimes B \rightarrow \mathbb{F}$ be an $H_{0}$-invariant pairing such that ${ }^{\perp} B=0$. If $B$ is simple, then $J_{a} \subset \operatorname{Ann}_{H_{0}^{*}} B$ for all $a \in A, a \neq 0$. Moreover, if $A$ is also simple, then $J_{a}=\operatorname{Ann}_{H_{0}^{*}} A$.

Proof. Let $a \in A, a \neq 0$ and take $f \in J_{a}$. It follows from (6-1) that for all $b \in B$, $0=\langle f \triangleright a, b\rangle=\langle a, f \triangleright b\rangle$. Thus, $\left\langle a, J_{a} \triangleright B\right\rangle=0$, hence $a \in^{\perp}\left(J_{a} \triangleright B\right)$. Since ${ }^{\perp} B=0$, this implies that $J_{a} \triangleright B$ is a proper $H_{0}^{*}$-submodule of $B$, hence $J_{a} \triangleright B=0$ by the simplicity of $B$.

Suppose now that $A$ is also simple. Then $J_{a}$ is a maximal left ideal for all $a \neq 0$. If $J_{a} \neq J_{a^{\prime}}$ for some $a, a^{\prime} \in A$ then $J_{a}+J_{a^{\prime}}=H_{0}^{*} \ni 1$, hence $B=0$, which contradicts the simplicity of $B$. Thus, $J_{a}=J_{a^{\prime}}$ for all $a, a^{\prime} \in A$ and so

$$
\operatorname{Ann}_{H_{0}^{*}} A=\bigcap_{a^{\prime} \in A} J_{a^{\prime}}=J_{a} .
$$

Since $A, B$ are simple and the form $\langle\cdot, \cdot\rangle$ is $H_{0}$-invariant and nonzero, ${ }^{\perp} B=0$ by Lemma 6.4. Then $\operatorname{Ann}_{H_{0}^{*}} A \subset \mathrm{Ann}_{H_{0}^{*}} B$ by Lemma 6.6. Let $R=H_{0}^{*} / \mathrm{Ann}_{H_{0}^{*}} A$. Then both $A$ and $B$ are $R$-modules in a natural way and are simple as such. Moreover, $A \cong B$ as $H_{0}$-comodules if and only if $A \cong B$ as $R$-modules. Furthermore, by 
definition of $R$ and Lemma 6.6 every nonzero element of $R$ acts on $A$ by an injective F-linear endomorphism. Since $A$ is a simple $H_{0}$-comodule, it is finite dimensional (see, e.g., [Montgomery 1993, Corollary 5.1.2]). Thus, each nonzero element of $R$ acts on $A$ by an $\mathbb{F}$-automorphism. This implies that $R$ is a division algebra, hence admits a unique, up to an isomorphism, simple finite dimensional module, and so $A \cong B$ as $R$-modules. Therefore, $A \cong B$ as objects in $\mathscr{C}$.

Remark 6.7. It can be shown that $R$ is a field, since for all $f, g \in H_{0}^{*}$ we have

$$
\langle f g \triangleright a, b\rangle=\langle g \triangleright a, f \triangleright b\rangle=\langle a,(g f) \triangleright b\rangle=\langle g f \triangleright a, b\rangle .
$$

Hence, since both $A$ and $B$ are simple, $f g-g f \in \mathrm{Ann}_{H_{0}} * A$.

Denote by $\mathscr{C}^{f}$ the full subcategory of $\mathscr{C}$ whose objects are direct sums of simple comodules with finite multiplicities. Thus, an object $V$ of $\mathscr{C}^{f}$ can be written as $V=\bigoplus_{i \in I} V_{i}$ where each $V_{i}$ is a finite direct sum of isomorphic simple subcomodules of $V$, and hence by [Montgomery 1995] is finite dimensional.

Lemma 6.8. Suppose that $V=\bigoplus_{i \in I} V_{i} \in \mathscr{C}^{f}$ admits an $H_{0}$-invariant bilinear form $\langle\cdot, \cdot\rangle: V \otimes V \rightarrow \mathbb{F}$. Then for any subobject $U$ of $V$ in $\mathscr{C}$,

$$
U^{\perp} \supset \bigoplus_{i \in \boldsymbol{I}} U_{i}^{\perp}
$$

where $U_{i}^{\perp}=\left\{v \in V_{i}:\left\langle U \cap V_{i}, v\right\rangle=0\right\}$.

Proof. By Proposition $6.5,\left\langle V_{\boldsymbol{i}}, V_{\boldsymbol{j}}\right\rangle=0$ if $\boldsymbol{i} \neq \boldsymbol{j}$. The assertion is now immediate.

\subsection{Quasiprimitive generators.}

Definition 6.9. Let $(A, \cdot, 1)$ be a unital algebra and $(B, \Delta, \varepsilon)$ be a coalgebra in $\mathscr{C}$. We say that an $H_{0}$-invariant pairing $\langle\cdot, \cdot\rangle: A \otimes B \rightarrow \mathbb{F}$ is compatible with $(A, \cdot, 1)$ and $(B, \Delta, \varepsilon)$ if

$$
\left\langle a \cdot a^{\prime}, b\right\rangle=\left\langle a \otimes a^{\prime}, \Delta(b)\right\rangle, \quad \varepsilon(b)=\langle 1, b\rangle
$$

for all $a, a^{\prime} \in A, b \in B$, where $\langle\cdot, \cdot\rangle:(A \otimes A) \otimes(B \otimes B) \rightarrow \mathbb{F}$ is defined by

$$
\left\langle a \otimes a^{\prime}, b \otimes b^{\prime}\right\rangle=\left\langle a, b^{\prime}\right\rangle\left\langle a^{\prime}, b\right\rangle .
$$

The main ingredient in our proof of Theorem 2.18 is the following result.

Theorem 6.10. Let $A$ be an algebra (denoted by $(A, \cdot, 1))$ and a coalgebra (denoted by $(A, \Delta, \varepsilon))$ in $\mathscr{C}^{f}$. Let $\langle\cdot, \cdot\rangle: A \otimes A \rightarrow \mathbb{F}$ be a compatible pairing between $(A, \cdot, 1)$ and $(A, \Delta, \varepsilon)$ satisfying $\langle a, a\rangle \neq 0$ for all $a \in A \backslash\{0\}$. Then $(A, \cdot, 1)$ is generated by $A_{1}=\operatorname{QPrim}(A, \Delta, \varepsilon)$. 
Proof. Let $B$ be a $\mathscr{C}$-subalgebra of $A$. Since $A \in \mathscr{C}^{f}$ and $B$ is its subobject, $A=\bigoplus_{i} A_{i}$ and $B=\bigoplus_{i} B_{i}$ where $B_{i}=A_{i} \cap B$. By Proposition 6.5, $\left\langle A_{i}, A_{j}\right\rangle=0$ for all $\boldsymbol{i} \neq \boldsymbol{j}$. We claim that $B^{\perp}$ is a coideal of $A$ in $\mathscr{C}$.

Indeed, for any $\boldsymbol{i}, \boldsymbol{j}$ we have

$$
\{0\}=\left\langle B_{\boldsymbol{i}} \cdot B_{j}, B^{\perp}\right\rangle=\left\langle B_{\boldsymbol{i}} \otimes B_{j}, \Delta\left(B^{\perp}\right)\right\rangle .
$$

Thus, $\Delta\left(B^{\perp}\right) \subset \bigoplus_{i, j}\left(B_{i} \otimes B_{j}\right)^{\perp}$ where $\left(B_{i} \otimes B_{j}\right)^{\perp}=\left\{z \in A_{j} \otimes A_{i}:\left\langle B_{i} \otimes B_{j}, z\right\rangle=0\right\}$. We need the following simple fact from linear algebra.

Lemma 6.11. Let $U, V$ be finite dimensional vector spaces over $\mathbb{F}$ and $U^{\prime} \subset U$, $V^{\prime} \subset V$ their subspaces. Then:

(a) $U^{\prime} \otimes V^{\prime}=\left(U^{\prime} \otimes V\right) \cap\left(U \otimes V^{\prime}\right)$;

(b) For any subspaces $V_{1}, V_{2}$ of $V$,

$$
\left(V_{1} \cap V_{2}\right)^{\perp}=V_{1}^{\perp}+V_{2}^{\perp}, \quad V_{1}^{\perp} \cap V_{2}^{\perp}=\left(V_{1}+V_{2}\right)^{\perp},
$$

where $W^{\perp}=\left\{f \in V^{*}: f(W)=0\right\}$ for any subspace $W \subset V$;

(c) $\left(U^{\prime} \otimes V^{\prime}\right)^{\perp}=V^{\prime \perp} \otimes U^{*}+V^{*} \otimes U^{\prime \perp}$, where we canonically identify $(U \otimes V)^{*}$ with $V^{*} \otimes U^{*}$.

Proof. Parts (a) and (b) are easily checked. For (c), note that $\left(U^{\prime} \otimes V\right)^{\perp}=V^{*} \otimes U^{\prime \perp}$ and $\left(U \otimes V^{\prime}\right)^{\perp}=V^{\prime \perp} \otimes U^{*}$. Hence by parts (a), and (b)

$$
\begin{aligned}
\left(U^{\prime} \otimes V^{\prime}\right)^{\perp} & =\left(\left(U^{\prime} \otimes V\right) \cap\left(U \otimes V^{\prime}\right)\right)^{\perp} \\
& =\left(U^{\prime} \otimes V\right)^{\perp}+\left(U \otimes V^{\prime}\right)^{\perp}=V^{*} \otimes U^{\prime \perp}+V^{\prime \perp} \otimes U^{*} .
\end{aligned}
$$

Since $A_{k}$ is finite dimensional and the restriction of $\langle\cdot, \cdot\rangle$ to $A_{k}$ is nondegenerate, we naturally identify $A_{\boldsymbol{k}}^{*}$ with $A_{\boldsymbol{k}}$ via $a \mapsto f_{a}$, where $f_{a}\left(a^{\prime}\right)=\left\langle a^{\prime}, a\right\rangle$. Then, applying Lemma 6.11(c) with $U=A_{i}, V=A_{j}, U^{\prime}=B_{i}$ and $V^{\prime}=B_{j}$, we obtain

$$
\left(B_{i} \otimes B_{j}\right)^{\perp}=A_{j} \otimes B_{i}^{\perp}+B_{j}^{\perp} \otimes A_{i},
$$

where $B_{k}^{\perp}=\left\{a \in A_{k}:\left\langle B_{k}, a\right\rangle=0\right\}$. We conclude that

$$
\Delta\left(B^{\perp}\right) \subset \bigoplus_{i, j}\left(B_{i} \otimes B_{j}\right)^{\perp} \subset A \otimes B^{\perp}+B^{\perp} \otimes A .
$$

To complete the proof of the claim, observe that $\varepsilon\left(B^{\perp}\right)=\left\langle 1, B^{\perp}\right\rangle=0$.

Now we complete the proof of Theorem 6.10. Let $B$ be the subalgebra of $A$ in $\mathscr{C}$ generated by the subobject $A_{1}=\operatorname{QPrim}_{\mathscr{C}}(A)$ of $A$, and suppose that $B \neq A$. Then, by the above claim, the orthogonal complement $I=B^{\perp}$ is a coideal of $A$ in $\mathscr{C}$. By Lemma 6.8, $I \supset \bigoplus_{i} B_{i}^{\perp} \neq\{0\}$ because $B_{i} \neq A_{i}$ for some $i$. Therefore, $I \neq\{0\}$ and so $I \cap A_{1} \neq\{0\}$ by Proposition 6.2. Yet $I \cap B=\{0\}$ since $\langle x, x\rangle \neq 0$ for all $x \in A$, hence $I \cap A_{1}=\{0\}$ and we obtain a contradiction. Thus, $B=A$. 
6.4. Proof of Theorems 1.2 and 2.18. Let $\mathbb{F}=\mathbb{Q}$ and define Green's pairing $\langle\cdot, \cdot\rangle: H_{\mathscr{A}} \otimes H_{\mathscr{A}} \rightarrow \mathbb{Q}$ (cf. [Green 1995]) by

$$
\langle[A],[B]\rangle=\frac{\delta_{[A],[B]}}{\left|\operatorname{Aut}_{\mathscr{A}}(A)\right|}
$$

for any $[A],[B] \in$ Iso $\mathscr{A}$.

Clearly, this pairing is positive definite and symmetric. We extend $\langle\cdot, \cdot\rangle$ to a symmetric bilinear form on $H_{\mathscr{A}} \otimes H_{\mathscr{A}}$ by

$$
\langle[A] \otimes[B],[C] \otimes[D]\rangle=\langle[A],[D]\rangle\langle[B],[C]\rangle
$$

for any $[A],[B],[C],[D] \in$ Iso $\mathscr{A}$.

Lemma 6.12. Let $\mathscr{A}$ be a cofinitary category. Then (6-3) is a compatible pairing (in the sense of Definition 6.9) between the Hall algebra $H_{\mathscr{A}}$ and the coalgebra $\left(H_{\mathscr{A}}, \Delta, \varepsilon\right)$.

Proof. We abbreviate $\Gamma=\Gamma_{\mathscr{A}}$ and let $\mathscr{C}=\mathscr{C}_{\Gamma}$ be the category of $\Gamma$-graded vector spaces or, equivalently, $\mathbb{Q} \Gamma$-comodules (cf. Example 6.3). It follows immediately from Example 6.3 that the pairing (6-3) is $\mathbb{Q} \Gamma$-invariant.

It remains to prove the compatibility in the sense of Definition 6.9, that is,

$$
\langle[A] \cdot[B],[C]\rangle=\langle[A] \otimes[B], \Delta([C])\rangle
$$

for all $[A],[B],[C] \in$ Iso $\mathscr{A}$. Indeed,

$$
\begin{aligned}
\langle[A] \cdot[B],[C]\rangle & =\frac{F_{A, B}^{C}}{\left|\operatorname{Aut}_{\mathscr{A}}(C)\right|}=\frac{F_{C}^{B, A}}{\left|\operatorname{Aut}_{\mathscr{A}}(B)\right|\left|\operatorname{Aut}_{\mathscr{A}}(A)\right|} \\
& =\sum_{\left[A^{\prime}\right],\left[B^{\prime}\right]} F_{C}^{B^{\prime}, A^{\prime}}\left\langle[A],\left[A^{\prime}\right]\right\rangle\left\langle[B],\left[B^{\prime}\right]\right\rangle \\
& =\sum_{\left[B^{\prime}\right],\left[A^{\prime}\right]} F_{C}^{B^{\prime}, A^{\prime}}\left\langle[A] \otimes[B],\left[B^{\prime}\right] \otimes\left[A^{\prime}\right]\right\rangle \\
& =\langle[A] \otimes[B], \Delta([C])\rangle .
\end{aligned}
$$

Proof of Theorems 1.2 and 2.18. Suppose that $\mathscr{A}$ is profinitary and cofinitary. Since for each $\gamma \in \Gamma=\Gamma_{\mathscr{A}},\left(H_{\mathscr{A}}\right)_{\gamma}$ is finite dimensional and hence is a finite direct sum of isomorphic simple left $\mathbb{Q} \Gamma$-comodules, $H_{\mathscr{A}} \in \mathscr{C}_{\Gamma}^{f}$. Then, clearly, $A=H_{\mathscr{A}}$ and the pairing (6-3) satisfy all the assumptions of Theorem 6.10. Therefore, $H_{\mathscr{A}}$ is generated by $A_{1}=\mathrm{QPrim}\left(H_{\mathscr{A}}, \Delta, \varepsilon\right)$ in $\mathscr{C}_{\Gamma}$.

Our next step is to show that $A_{1}=\operatorname{Prim}\left(H_{\mathscr{A}}, \Delta, \varepsilon\right)$, which gives the first assertion of Main Theorem 1.2. For that, we need the following result.

Lemma 6.13. Let $C=\bigoplus_{\gamma \in \Gamma} C_{\gamma}$ be a coalgebra in the category $\mathscr{C}_{\Gamma}$. Assume that for every $\gamma \in \Gamma^{+}$, there exists $h_{\gamma} \in \mathbb{Z}_{>0}$ such that $\gamma$ cannot be written as a sum of 
more than $h_{\gamma}$ elements of $\Gamma^{+}$. Then $\operatorname{Corad}_{\mathscr{C}}(C) \subset C_{\mathbf{0}}$ where $\mathbf{0}$ is the zero element of $\Gamma$.

Proof. First, observe that $\mathbf{0}$ is the only invertible element of $\Gamma$, since otherwise $\mathbf{0}=\alpha+\beta$ for some $\alpha, \beta \in \Gamma^{+}$and so $\alpha=(n+1) \alpha+n \beta$ for any $n \in \mathbb{Z}_{>0}$, which is a contradiction. Since for any subcoalgebra $D=\bigoplus_{\gamma \in \Gamma} D_{\gamma}$ of $C$ in $\mathscr{C}_{\Gamma}$

$$
\Delta\left(D_{\gamma}\right) \subset \bigoplus_{\gamma^{\prime}, \gamma^{\prime \prime} \in \Gamma: \gamma=\gamma^{\prime}+\gamma^{\prime \prime}} D_{\gamma^{\prime}} \otimes D_{\gamma^{\prime \prime}}
$$

it follows that $\Delta\left(D_{0}\right) \subset D_{0} \otimes D_{0}$. Therefore, $D_{0}$ is a subcoalgebra of $D$.

We claim that $D=0$ if and only if $D_{\mathbf{0}}=0$. Indeed, if $D_{\mathbf{0}}=0$, since for the $k$-th iterated comultiplication $\Delta^{k}$ we have

$$
\Delta^{k}\left(D_{\gamma}\right) \subset \sum_{\gamma_{0}, \ldots, \gamma_{k} \in \Gamma: \gamma_{0}+\cdots+\gamma_{k}=\gamma} D_{\gamma_{0}} \otimes \cdots \otimes D_{\gamma_{k}},
$$

it follows that $\Delta^{h_{\gamma}}\left(D_{\gamma}\right)=0$, since then in each summand we must have $\gamma_{i}=0$ for some $0 \leq i \leq h_{\gamma}$ by the assumptions of the lemma. This implies that $D_{\gamma}=0$ for all $\gamma \in \Gamma$, hence $D=0$. The converse is obvious.

Thus, if $D$ is a simple subcoalgebra of $C$, then $D_{\mathbf{0}} \neq 0$ and so $D=D_{\mathbf{0}}$.

By Lemma 5.6, $\Gamma_{\mathscr{A}}$ satisfies the assumptions of Lemma 6.13, with $h_{\gamma} \leq s_{\gamma}$, hence $\operatorname{Corad}_{\mathscr{C}}\left(H_{\mathscr{A}}\right)=\mathbb{Q}$ and $\operatorname{QPrim}_{\mathscr{C}}\left(H_{\mathscr{A}}\right)=\mathbb{Q} \oplus \operatorname{Prim}\left(H_{\mathscr{A}}\right)$. This proves the first assertion of Main Theorem 1.2. It remains to prove the second assertion (and thus complete the proof of Theorem 2.18), namely, that $\operatorname{Prim}\left(H_{\mathscr{A}}\right)$ is a minimal generating space of $H_{\mathscr{A}}$. We need the following result.

Lemma 6.14. Suppose $A$ is both a unital algebra and coalgebra with $\Delta(1)=1 \otimes 1$. Assume that $A$ admits a compatible pairing $\langle\cdot, \cdot\rangle: A \otimes A \rightarrow \mathbb{F}$, in the sense of Definition 6.9, such that $\langle a, 1\rangle=\varepsilon(a)$ for all $a \in A$. Let $V=\operatorname{Prim}(A)$. Then $1 \notin V$ and $\left\langle\sum_{k \geq 2} V^{k}, \mathbb{F} \oplus V\right\rangle=0$.

Proof. Since $v \in V$ is primitive, $\varepsilon(v)=0$. Furthermore, we show that $\varepsilon: A \rightarrow \mathbb{F}$ is a homomorphism of algebras. Indeed, given $a, a^{\prime} \in A$, we have

$$
\varepsilon\left(a a^{\prime}\right)=\left\langle a a^{\prime}, 1\right\rangle=\left\langle a \otimes a^{\prime}, \Delta(1)\right\rangle=\left\langle a \otimes a^{\prime}, 1 \otimes 1\right\rangle=\left\langle a^{\prime}, 1\right\rangle\langle a, 1\rangle=\varepsilon(a) \varepsilon\left(a^{\prime}\right) .
$$

This immediately implies that $\varepsilon\left(V^{\ell}\right)=0$ and $\left\langle V^{\ell}, V^{0}\right\rangle=0, \ell>0$. Finally, let $v \in V$ and $x, y \in \operatorname{ker} \varepsilon$. Then

(6-4) $\langle x y, v\rangle=\langle x \otimes y, \Delta(v)\rangle=\langle x \otimes y, v \otimes 1+1 \otimes v\rangle=\langle y, v\rangle \varepsilon(x)+\varepsilon(y)\langle x, v\rangle=0$.

Let $\ell>1$. Since $V^{\ell} \subset V \cdot V^{\ell-1}$ and $V^{k} \subset \operatorname{ker} \varepsilon$ for all $k>0$, it follows that $\left\langle V^{\ell}, V\right\rangle=0$. 
Let $V_{\mathscr{A}}=\operatorname{Prim}\left(H_{\mathscr{A}}\right)$ and $\left(H_{\mathscr{A}}\right)_{>1}=\sum_{r \geq 2} V_{\mathscr{A}}^{r}$. From Lemma 6.14, we have $\left\langle\left(H_{\mathscr{A}}\right)_{>1}, \mathbb{Q} \oplus V_{\mathscr{A}}\right\rangle=0$. Since the pairing $\langle\cdot, \cdot\rangle$ on $H_{\mathscr{A}}$ is symmetric positive definite, $\left(H_{\mathscr{A}}\right)_{>1} \cap\left(\mathbb{Q} \oplus V_{\mathscr{A}}\right)=\{0\}$, hence the sum $\left(\mathbb{Q} \oplus V_{\mathscr{A}}\right)+\left(H_{\mathscr{A}}\right)_{>1}$ is direct. This proves the second assertion of Main Theorem 1.2 and completes the proof of Theorem 2.18 .

\subsection{Proof of Corollary 2.19 and estimates for primitive elements.}

Proof of Corollary 2.19. Let $H_{\mathscr{A}}^{+}=\operatorname{ker} \varepsilon$ and let $R \subset H_{\mathscr{A}}^{+}$be a generating space for $H_{\mathscr{A}}$. Then $\left(H_{\mathscr{A}}^{+}\right)^{\ell}=\sum_{k>\ell} R^{k}, \ell \geq 1$. Taking $R=\mathbb{Q}$ Ind $\mathscr{A}$ (Theorem 1.1) and $R=\operatorname{Prim}\left(H_{\mathscr{A}}\right)$ (Theorem 2.18) we conclude that

$$
P=\left(H_{\mathscr{A}}^{+}\right)^{2}=\sum_{k \geq 2} \operatorname{Prim}\left(H_{\mathscr{A}}\right)^{k}=\sum_{k \geq 2}(\mathbb{Q} \text { Ind } \mathscr{A})^{k} .
$$

On the other hand, $H_{\mathscr{A}}^{+}=\operatorname{Prim}\left(H_{\mathscr{A}}\right)+P$ and $P \cap \operatorname{Prim}\left(H_{\mathscr{A}}\right)=\{0\}$ by Lemma 6.14. Therefore, $H_{\mathscr{A}}^{+}=\operatorname{Prim}\left(H_{\mathscr{A}}\right) \oplus P$. The graded version is immediate.

Proof of Proposition 2.20 and Lemma 2.21. We need the following obvious fact from linear algebra.

Lemma 6.15. Let $U$ be a finite dimensional $\mathbb{F}$-vector space and $U_{1}, U_{1}^{\prime}, U_{2}$ be subspaces of $U$ such that $U=U_{1}+U_{2}=U_{1}^{\prime}+U_{2}$. If $U_{1} \cap U_{2}=\{0\}$, then $\operatorname{dim}_{\mathbb{F}} U_{1}=\operatorname{dim}_{\mathbb{F}} U_{1}^{\prime}-\operatorname{dim}_{\mathbb{F}}\left(U_{1}^{\prime} \cap U_{2}\right)$.

Taking into account Corollary 2.19, we apply this lemma with $U=\left(H_{\mathscr{A}}\right)_{\gamma}$, $U_{1}^{\prime}=\mathbb{Q}$ Ind $\mathscr{A}_{\gamma}, U_{2}=P_{\gamma}$ and $U_{1}=\operatorname{Prim}\left(H_{\mathscr{A}}\right)_{\gamma}$ to obtain

$$
\operatorname{dim}_{\mathbb{Q}} \operatorname{Prim}\left(H_{\mathscr{A}}\right)_{\gamma}=\# \text { Ind } \mathscr{A}_{\gamma}-\operatorname{dim}_{\mathbb{Q}}\left(P_{\gamma} \cap \mathbb{Q} \text { Ind } \mathscr{A}_{\gamma}\right),
$$

which yields Proposition 2.20.

To prove Lemma 2.21 , note that $\mathbb{Q}($ Iso $\mathscr{A} \backslash$ Ind $\mathscr{A})=(\mathbb{Q} \text { Ind } \mathscr{A})^{\perp}$. Thus,

$$
\begin{aligned}
\operatorname{Prim}\left(H_{\mathscr{A}}\right)_{\gamma} \cap \mathbb{Q}(\text { Iso } \mathscr{A} \backslash \text { Ind } \mathscr{A}) & \subset\left(\mathbb{Q} \text { Ind } \mathscr{A}_{\gamma}\right)^{\perp} \cap P_{\gamma}^{\perp} \\
& =\left(\mathbb{Q} \text { Ind } \mathscr{A}_{\gamma}+P_{\gamma}\right)^{\perp}=\left(H_{\mathscr{A}}\right)_{\gamma}^{\perp}=0
\end{aligned}
$$

by Lemma 6.11(b) and Corollary 2.19 .

\section{Proof of Theorem 2.26}

7.1. Diagonally braided categories. We call a bialgebra $H_{0}$ coquasitriangular if it has a skew Hopf self-pairing $\mathcal{R}: H_{0} \otimes H_{0} \rightarrow \mathbb{Q}$. Let $\mathscr{C}$ be the category of left $H_{0}$-comodules. This category is braided via the commutativity constraint $\Psi_{U, V}: U \otimes V \rightarrow V \otimes U$ for all objects $U, V$ of $\mathscr{C}$ defined by

$$
\Psi_{U, V}(u \otimes v)=\mathcal{R}\left(u^{(-1)}, v^{(-1)}\right) \cdot v^{(0)} \otimes u^{(0)}
$$


for all $u \in U, v \in V$, where we use the Sweedler-like notation for the coactions $\delta_{U}(u)=u^{(-1)} \otimes u^{(0)}$ and $\delta_{V}(v)=v^{(-1)} \otimes v^{(0)}$. We will write $\mathscr{C}_{\mathcal{R}}$ to emphasize that $\mathscr{C}$ is a braided category.

Remark 7.1. The category $\mathscr{C}_{\chi}$ introduced in Lemma 2.22 is equivalent to the category of $H_{0}$-comodules, where $H_{0}=\mathbb{Q} \Gamma$ is the monoidal algebra of $\Gamma$ and $\left.\mathcal{R}\right|_{\Gamma \times \Gamma}=\chi$.

Our present aim is to prove the following result.

Theorem 7.2. Let $B$ be a bialgebra in $\mathscr{C}_{\mathcal{R}}$.

(a) The space $V=\operatorname{Prim}(B)$ is a subobject of $B$ in $\mathscr{C}_{\mathcal{R}}$.

(b) Suppose that B admits a compatible pairing, in the sense of Definition 6.9, such that $\langle b, 1\rangle=\varepsilon(b)$ and $\langle b, b\rangle \neq 0$ for all $b \in B \backslash\{0\}$. Then the canonical inclusion $V \hookrightarrow B$ extends to an injective homomorphism

$$
\mathcal{B}(V) \rightarrow B
$$

of bialgebras in $\mathscr{C}_{\mathcal{R}}$. In particular, if $B$ is generated by $V$, then (7-1) is an isomorphism.

Proof. Part (a) is a special case of the following simple fact.

Lemma 7.3. If $C$ is a coalgebra in $\mathscr{C}_{\mathcal{R}}$ with unity, then $V:=\operatorname{Prim}(C)$ is a subobject of $C$ in $\mathscr{C}_{\mathcal{R}}$.

Proof. Denote by $\delta_{C}: C \rightarrow H_{0} \otimes C$ the left coaction of $H_{0}$ on $C$. All we have to show is that $\delta_{C}(V) \subset H_{0} \otimes V$. Fix a basis $\left\{b_{i}\right\}$ of $H_{0}$ and let $v \in \operatorname{Prim}(C)$. Write

$$
\delta_{C}(v)=\sum_{i} b_{i} \otimes v_{i}, \quad v_{i} \in C .
$$

Since $\Delta: C \rightarrow C \otimes C$ is a morphism of left $H_{0}$-comodules,

$$
(1 \otimes \Delta) \circ \delta_{C}(v)=\delta_{C}(v \otimes 1)+\delta_{C}(1 \otimes v) .
$$

Taking into account that $\delta_{C}(1)=1 \otimes 1$, we obtain

$$
\sum_{i} b_{i} \otimes \Delta\left(v_{i}\right)=\sum_{i} b_{i} \otimes v_{i} \otimes 1+\sum_{i} b_{i} \otimes 1 \otimes v_{i},
$$

which implies that

$$
\Delta\left(v_{i}\right)=v_{i} \otimes 1+1 \otimes v_{i},
$$

that is, $v_{i} \in V$ for all $i$.

Now we prove (b). Denote by $B^{\prime}$ the subalgebra of $B$ generated by $V=\operatorname{Prim}(B)$. It is sufficient to show that $B^{\prime}=\mathcal{B}(V)$. We need the following result.

Proposition 7.4. $B^{\prime}=\bigoplus_{k \geq 0} V^{k}$, hence $B^{\prime}$ is a graded algebra. 
Proof. We prove that $\left\langle V^{\ell}, V^{k}\right\rangle=0$ for all $0 \leq k<\ell$ by induction on the pairs $(k, \ell)$ with $k<\ell$, ordered lexicographically. The induction base for $k=0,1$ is established in Lemma 6.14. Now, fix $\ell>2$ and suppose that $\left\langle V^{s}, V^{r}\right\rangle=0$ for all $r<s<\ell$. Let $1<k<\ell$. Since $\Delta$ is a homomorphism of algebras,

$$
\Delta\left(V^{k}\right) \subset(V \otimes 1+1 \otimes V)^{k} \subset \sum_{i=0}^{k} V^{k-i} \otimes V^{i},
$$

hence

$$
\begin{aligned}
\left\langle V^{\ell}, V^{k}\right\rangle & \subset\left\langle V \otimes V^{\ell-1}, \Delta\left(V^{k}\right)\right\rangle \subset \sum_{i=0}^{k}\left\langle V \otimes V^{\ell-1}, V^{k-i} \otimes V^{i}\right\rangle \\
& =\sum_{i=0}^{k}\left\langle V, V^{i}\right\rangle\left\langle V^{\ell-1}, V^{k-i}\right\rangle=\langle V, V\rangle\left\langle V^{\ell-1}, V^{k-1}\right\rangle=\{0\}
\end{aligned}
$$

by the inductive hypothesis. It remains to show that the sum $\sum_{k \geq 0} V^{k}$ is direct, which is an immediate consequence of the following obvious fact.

Lemma 7.5. Let $U_{i}, i \in \mathbb{Z}_{\geq 0}$, be subspaces of an $\mathbb{F}$-vector space $U$ with a bilinear form $\langle\cdot, \cdot\rangle: U \otimes U \rightarrow \mathbb{F}$ such that $\left\langle U_{j}, U_{i}\right\rangle=0$ if $j>i$ and $\langle u, u\rangle \neq 0$ for all $u \in U \backslash\{0\}$. Then the sum $\sum_{i} U_{i}$ is direct.

This completes the proof of Proposition 7.4.

Since $B_{0}^{\prime}=\mathbb{Q}$ and $B_{1}^{\prime}=V=\operatorname{Prim}\left(B^{\prime}\right)=\operatorname{Prim}(B), B^{\prime}$ is the Nichols algebra of $V$ by Definition 2.23. Theorem 7.2 is therefore proved.

7.2. Proof of Theorem 2.26. We need the following reformulation of Green's celebrated theorem for Hall algebras ([Green 1995]; see also [Walker 2011]).

Proposition 7.6. Let $\mathscr{A}$ be a finitary and cofinitary hereditary abelian category. Then the Hall algebra $H_{\mathscr{A}}$ is a bialgebra in $\mathscr{C}_{\chi_{\mathscr{A}}}$ with the coproduct $\Delta$ given by (2-3) and the counit $\varepsilon$ given by (2-4).

Proof. For every $[C],\left[C^{\prime}\right] \in$ Iso $\mathscr{A}$ we have

$$
\begin{aligned}
\Delta([C]) \Delta\left(\left[C^{\prime}\right]\right) & =\left(\sum_{[A],[B]} F_{C}^{A, B} \cdot[A] \otimes[B]\right)\left(\sum_{\left[A^{\prime}\right],\left[B^{\prime}\right]} F_{C^{\prime}}^{A^{\prime}, B^{\prime}} \cdot\left[A^{\prime}\right] \otimes\left[B^{\prime}\right]\right) \\
& =\sum_{[A],[B],\left[A^{\prime}\right],\left[B^{\prime}\right]} F_{C}^{A, B} F_{C^{\prime}}^{A^{\prime}, B^{\prime}} \cdot \frac{\left|\operatorname{Ext}_{\mathscr{A}}^{1}\left(B, A^{\prime}\right)\right|}{\left|\operatorname{Hom}_{\mathscr{A}}\left(B, A^{\prime}\right)\right|}[A]\left[A^{\prime}\right] \otimes[B]\left[B^{\prime}\right] \\
& =\sum_{\substack{[A],[B],\left[A^{\prime}\right],\left[B^{\prime}\right],\left[A^{\prime \prime}\right],\left[B^{\prime \prime}\right]}} F_{C}^{A, B} F_{C^{\prime}}^{A^{\prime}, B^{\prime}} F_{A, A^{\prime}}^{A^{\prime \prime}} F_{B, B^{\prime}}^{B^{\prime \prime}} \frac{\left|\operatorname{Ext}_{\mathscr{A}}^{1}\left(B, A^{\prime}\right)\right|}{\left|\operatorname{Hom}_{\mathscr{A}}\left(B, A^{\prime}\right)\right|} \cdot\left[A^{\prime \prime}\right] \otimes\left[B^{\prime \prime}\right]
\end{aligned}
$$


On the other hand,

$$
\Delta\left([C]\left[C^{\prime}\right]\right)=\sum_{\left[C^{\prime \prime}\right]} F_{C, C^{\prime}}^{C^{\prime \prime}} \Delta\left(\left[C^{\prime \prime}\right]\right)=\sum_{\left[C^{\prime \prime}\right],\left[A^{\prime \prime}\right],\left[B^{\prime \prime}\right]} F_{C, C^{\prime}}^{C^{\prime \prime}} F_{C^{\prime \prime}}^{A^{\prime \prime}, B^{\prime \prime}} \cdot\left[A^{\prime \prime}\right] \otimes\left[B^{\prime \prime}\right] .
$$

We need the following lemma.

Lemma 7.7 ([Green 1995, Theorem 2], see also [Schiffmann 2012]). If $\mathscr{A}$ is a finitary and cofinitary hereditary abelian category, then for any objects $A^{\prime \prime}, B^{\prime \prime}, C, C^{\prime}$ of $\mathscr{A}$ one has

$$
\begin{aligned}
\sum_{[A],\left[A^{\prime}\right],[B],\left[B^{\prime}\right] \in \operatorname{Iso} \mathscr{A}} \frac{\left|\operatorname{Ext}_{\mathscr{A}}^{1}\left(B, A^{\prime}\right)\right|}{\left|\operatorname{Hom}_{\mathscr{A}}\left(B, A^{\prime}\right)\right|} \cdot F_{B, B^{\prime}}^{B^{\prime \prime}} F_{A, A^{\prime}}^{A^{\prime \prime}} F_{C}^{A, B} F_{C^{\prime}}^{A^{\prime}, B^{\prime}} & \\
& =\sum_{\left[C^{\prime \prime}\right] \in \operatorname{Iso} \mathscr{A}} F_{C, C^{\prime}}^{C^{\prime \prime}} F_{C^{\prime \prime}}^{A^{\prime \prime}, B^{\prime \prime}}
\end{aligned}
$$

This immediately implies that $\Delta([C]) \Delta\left(\left[C^{\prime}\right]\right)=\Delta\left([C]\left[C^{\prime}\right]\right)$.

Theorem 2.26 now follows from Proposition 7.6 and Theorem 7.2.

\section{Acknowledgments}

We are grateful to Bernhard Keller, Dylan Ruppel and Vadim Vologodsky for stimulating discussions. An important part of this work was done during Berenstein's visit to the MSRI in the framework of the "Cluster algebras" program and he thanks the Institute and the organizers for their hospitality and support. Greenstein is indebted to the Department of Mathematics of Uppsala University and especially Volodymyr Mazorchuk for their hospitality and support. In the final stage of work on this paper we benefited from the hospitality of Institut des Hautes Études Scientiques (IHES), which we gratefully acknowledge.

\section{References}

[Andruskiewitsch and Schneider 2002] N. Andruskiewitsch and H.-J. Schneider, "Pointed Hopf algebras", pp. 1-68 in New directions in Hopf algebras, edited by S. Montgomery and H.-J. Schneider, Mathematical Sciences Research Institute Publications 43, Cambridge University Press, 2002. MR 2003e:16043 Zbl 1011.16025

[Auslander et al. 1995] M. Auslander, I. Reiten, and S. O. Smalø, Representation theory of Artin algebras, Cambridge Studies in Advanced Mathematics 36, Cambridge University Press, 1995. MR 96c:16015 Zbl 0834.16001

[Baumann and Kassel 2001] P. Baumann and C. Kassel, "The Hall algebra of the category of coherent sheaves on the projective line", J. Reine Angew. Math. 533 (2001), 207-233. MR 2002g:14019 Zbl 0967.18005

[Berenstein and Greenstein 2013] A. Berenstein and J. Greenstein, "Quantum Chevalley groups", pp. 71-102 in Noncommutative birational geometry, representations and combinatorics, edited by 
A. Berenstein and V. Retakh, Contemporary Mathematics 592, American Mathematical Society, Providence, RI, 2013. MR 3087940 Zbl 1321.18006

[Buchsbaum 1959] D. A. Buchsbaum, "A note on homology in categories", Ann. of Math. (2) 69 (1959), 66-74. MR 25 \#3974 Zbl 0084.26801

[Bühler 2010] T. Bühler, "Exact categories”, Expo. Math. 28:1 (2010), 1-69. MR 2011e:18020 Zbl 1192.18007

[Burban and Schiffmann 2012] I. Burban and O. Schiffmann, "On the Hall algebra of an elliptic curve, I”, Duke Math. J. 161:7 (2012), 1171-1231. MR 2922373 Zbl 1286.16029

[Deng and Ruan 2015] B. Deng and S. Ruan, "Hall polynomials for tame type", preprint, 2015. arXiv 1512.03504

[Deng and Xiao 2003] B. Deng and J. Xiao, "A new approach to Kac's theorem on representations of valued quivers", Math. Z. 245:1 (2003), 183-199. MR 2004k:16032 Zbl 1053.16010

[Dlab and Ringel 1976] V. Dlab and C. M. Ringel, Indecomposable representations of graphs and algebras, Memoirs of the American Mathematical Society 6:173, American Mathematical Society, Providence, RI, 1976. MR 56 \#5657 Zbl 0332.16015

[Gabriel 1973] P. Gabriel, "Indecomposable representations, II", pp. 81-104 in Symposia Mathematica, XI: Convegno di Algebra Commutativa, INDAM (Rome, 1971), Academic Press, London, 1973. MR 49 \#5132 Zbl 0276.16001

[Green 1995] J. A. Green, "Hall algebras, hereditary algebras and quantum groups", Invent. Math. 120:2 (1995), 361-377. MR 96c:16016 Zbl 0836.16021

[Guo and Peng 1997] J. Y. Guo and L. Peng, "Universal PBW-basis of Hall-Ringel algebras and Hall polynomials", J. Algebra 198:2 (1997), 339-351. MR 99b:16033 Zbl 0893.16005

[Hausel 2010] T. Hausel, "Kac's conjecture from Nakajima quiver varieties", Invent. Math. 181:1 (2010), 21-37. MR 2011d:14033 Zbl 1198.16016

[Hua 2000] J. Hua, "Counting representations of quivers over finite fields", J. Algebra 226:2 (2000), 1011-1033. MR 2001j:16017 Zbl 0972.16006

[Hubery 2004] A. Hubery, "The composition algebra of an affine quiver", preprint, 2004. arXiv math/ 0403206

[Hubery 2005] A. Hubery, "Symmetric functions and the centre of the Ringel-Hall algebra of a cyclic quiver", Math. Z. 251:3 (2005), 705-719. MR 2006i:16019 Zbl 1084.16010

[Hubery 2006] A. Hubery, "From triangulated categories to Lie algebras: a theorem of Peng and Xiao", pp. 51-66 in Trends in representation theory of algebras and related topics, edited by J. A. de la Peña and R. Bautista, Contemporary Mathematics 406, American Mathematical Society, Providence, RI, 2006. MR 2008f:16033 Zbl 1107.16021

[Hubery 2007] A. Hubery, "Ringel-Hall algebras", lecture notes, 2007, available at http://wwwmath. uni-paderborn.de/ hubery/RHAlg.pdf.

[Joyce 2006] D. Joyce, "Configurations in abelian categories, I: Basic properties and moduli stacks", Adv. Math. 203:1 (2006), 194-255. MR 2007b:14023 Zbl 1102.14009

[Joyce 2007] D. Joyce, "Configurations in abelian categories, II: Ringel-Hall algebras", Adv. Math. 210:2 (2007), 635-706. MR 2008f:14022 Zbl 1119.14005

[Kac 1980] V. G. Kac, "Infinite root systems, representations of graphs and invariant theory", Invent. Math. 56:1 (1980), 57-92. MR 82j:16050 Zbl 0427.17001 
[Kapranov 1997] M. M. Kapranov, "Eisenstein series and quantum affine algebras", in Algebraic geometry, 7, Itogi Nauki i Tekhniki, Seriya Sovremennaya Matematika i Ee Prilozheniya. Tematicheskie Obzory 41, VINITI, Moscow, 1997. Translated in J. Math. Sci (New York) 84:5 (1997), 1311-1360. MR 98i:11033 Zbl 0929.11015

[Kapranov et al. 2012] M. M. Kapranov, O. Schiffmann, and E. Vasserot, "The Hall algebra of a curve", preprint, 2012. arXiv 1201.6185v2

[Keller 1990] B. Keller, "Chain complexes and stable categories”, Manuscripta Math. 67:4 (1990), 379-417. MR 91h:18006 Zbl 0753.18005

[Macdonald 1979] I. G. Macdonald, Symmetric functions and Hall polynomials, Clarendon/Oxford University Press, New York, 1979. MR 84g:05003 Zbl 0487.20007

[Montgomery 1993] S. Montgomery, Hopf algebras and their actions on rings, CBMS Regional Conference Series in Mathematics 82, American Mathematical Society, Providence, RI, 1993. MR 94i:16019 Zbl 0793.16029

[Montgomery 1995] S. Montgomery, "Indecomposable coalgebras, simple comodules, and pointed Hopf algebras", Proc. Amer. Math. Soc. 123:8 (1995), 2343-2351. MR 95j:16046 Zbl 0836.16024

[Polishchuk and Positselski 2005] A. Polishchuk and L. Positselski, Quadratic algebras, University Lecture Series 37, American Mathematical Society, Providence, RI, 2005. MR 2006f:16043 Zbl 1145.16009

[Quillen 1973] D. Quillen, "Higher algebraic K-theory, I", pp. 85-147 in Algebraic K-theory, I: Higher K-theories (Seattle, WA, 1972), edited by H. Bass, Lecture Notes in Mathematics 341, Springer, Berlin, 1973. MR 49 \#2895 Zbl 0292.18004

[Riedtmann 1994] C. Riedtmann, "Lie algebras generated by indecomposables", J. Algebra 170:2 (1994), 526-546. MR 96e:16013 Zbl 0841.16018

[Ringel 1990a] C. M. Ringel, "Hall algebras”, pp. 433-447 in Topics in algebra, part 1 (Warsaw, 1988), edited by S. Balcerzyk et al., Banach Center Publications 26:1, PWN, Warsaw, 1990. MR 93f:16027 Zbl 0778.16004

[Ringel 1990b] C. M. Ringel, "Hall algebras and quantum groups", Invent. Math. 101:3 (1990), 583-591. MR 91i:16024 Zbl 0735.16009

[Schiffmann 2012] O. Schiffmann, "Lectures on Hall algebras", pp. 1-141 in Geometric methods in representation theory, II (Grenoble, 2008), edited by M. Brion, Séminaires et Congrès 24, part 2, Société Mathématique de France, Paris, 2012. MR 3202707 Zbl 1309.18012 arXiv math.RT/0611617

[Sevenhant and Van Den Bergh 2001] B. Sevenhant and M. Van Den Bergh, "A relation between a conjecture of Kac and the structure of the Hall algebra", J. Pure Appl. Algebra 160:2-3 (2001), 319-332. MR 2002f:17031 Zbl 1025.17006

[Sweedler 1969] M. E. Sweedler, Hopf algebras, W. A. Benjamin, New York, 1969. MR 40 \#5705 Zbl 0194.32901

[Szántó 2006] C. Szántó, "Hall numbers and the composition algebra of the Kronecker algebra", Algebr. Represent. Theory 9:5 (2006), 465-495. MR 2007g:16024 Zbl 1161.16011

[Walker 2011] C. D. Walker, A categorification of Hall algebras, thesis, University of California, Riverside, 2011, available at http://search.proquest.com/docview/883977400. MR 2912198

[Yoneda 1954] N. Yoneda, "On the homology theory of modules", J. Fac. Sci. Univ. Tokyo. Sect. I. 7 (1954), 193-227. MR 16,947d Zbl 0058.01902

[Zelevinsky 1981] A. V. Zelevinsky, Representations of finite classical groups: a Hopf algebra approach, Lecture Notes in Mathematics 869, Springer, Berlin, 1981. MR 83k:20017 Zbl 0465.20009 
Received March 23, 2015.

ARKADY BERENSTEIN

DEPARTMENT OF MATHEMATICS

UNIVERSITY OF OREGON

EUGENE, OR 97403-1222

UNITED STATES

arkadiy@uoregon.edu

\section{JACOB GREENSTEIN}

DEPARTMENT OF MATHEMATICS

UNIVERSITY OF CALIFORNIA

RIVERSIDE, CA 92521-0135

UNITED STATES

jacob.greenstein@ucr.edu 


\title{
PACIFIC JOURNAL OF MATHEMATICS
}

\author{
msp.org/pjm
}

Founded in 1951 by E. F. Beckenbach (1906-1982) and F. Wolf (1904-1989)

\section{EDITORS}

Don Blasius (Managing Editor)

Department of Mathematics

University of California

Los Angeles, CA 90095-1555

blasius@math.ucla.edu

\author{
Paul Balmer \\ Department of Mathematics \\ University of California \\ Los Angeles, CA 90095-1555 \\ balmer@math.ucla.edu \\ Robert Finn \\ Department of Mathematics \\ Stanford University \\ Stanford, CA 94305-2125 \\ finn@math.stanford.edu \\ Sorin Popa \\ Department of Mathematics \\ University of California \\ Los Angeles, CA 90095-1555 \\ popa@math.ucla.edu
}

\author{
Vyjayanthi Chari \\ Department of Mathematics \\ University of California \\ Riverside, CA 92521-0135 \\ chari@math.ucr.edu \\ Kefeng Liu \\ Department of Mathematics \\ University of California \\ Los Angeles, CA 90095-1555 \\ liu@math.ucla.edu \\ Jie Qing \\ Department of Mathematics \\ University of California \\ Santa Cruz, CA 95064 \\ qing@ cats.ucsc.edu
}

\section{PRODUCTION}

Silvio Levy, Scientific Editor, production@msp.org

\section{SUPPORTING INSTITUTIONS}

ACADEMIA SINICA, TAIPEI

CALIFORNIA INST. OF TECHNOLOGY

INST. DE MATEMÁTICA PURA E APLICADA

KEIO UNIVERSITY

MATH. SCIENCES RESEARCH INSTITUTE

NEW MEXICO STATE UNIV.

OREGON STATE UNIV.

\author{
STANFORD UNIVERSITY \\ UNIV. OF BRITISH COLUMBIA \\ UNIV. OF CALIFORNIA, BERKELEY \\ UNIV. OF CALIFORNIA, DAVIS \\ UNIV. OF CALIFORNIA, LOS ANGELES \\ UNIV. OF CALIFORNIA, RIVERSIDE \\ UNIV. OF CALIFORNIA, SAN DIEGO \\ UNIV. OF CALIF., SANTA BARBARA
}

\author{
Daryl Cooper \\ Department of Mathematics \\ University of California \\ Santa Barbara, CA 93106-3080 \\ cooper@math.ucsb.edu \\ Jiang-Hua Lu \\ Department of Mathematics \\ The University of Hong Kong \\ Pokfulam Rd., Hong Kong \\ jhlu@maths.hku.hk \\ Paul Yang \\ Department of Mathematics \\ Princeton University \\ Princeton NJ 08544-1000 \\ yang@math.princeton.edu
}

These supporting institutions contribute to the cost of publication of this Journal, but they are not owners or publishers and have no responsibility for its contents or policies.

See inside back cover or msp.org/pjm for submission instructions.

The subscription price for 2016 is US $\$ 440 /$ year for the electronic version, and $\$ 600 /$ year for print and electronic.

Subscriptions, requests for back issues and changes of subscribers address should be sent to Pacific Journal of Mathematics, P.O. Box 4163, Berkeley, CA 94704-0163, U.S.A. The Pacific Journal of Mathematics is indexed by Mathematical Reviews, Zentralblatt MATH, PASCAL CNRS Index, Referativnyi Zhurnal, Current Mathematical Publications and Web of Knowledge (Science Citation Index).

The Pacific Journal of Mathematics (ISSN 0030-8730) at the University of California, c/o Department of Mathematics, 798 Evans Hall \#3840, Berkeley, CA 94720-3840, is published twelve times a year. Periodical rate postage paid at Berkeley, CA 94704, and additional mailing offices. POSTMASTER: send address changes to Pacific Journal of Mathematics, P.O. Box 4163, Berkeley, CA 94704-0163.

PJM peer review and production are managed by EditFLOW ${ }^{\circledR}$ from Mathematical Sciences Publishers.

\section{PUBLISHED BY}

\section{mathematical sciences publishers \\ nonprofit scientific publishing}

http://msp.org/

(C) 2016 Mathematical Sciences Publishers 


\title{
PACIFIC JOURNAL OF MATHEMATICS
}

\author{
Volume $281 \quad$ No. $2 \quad$ April 2016
}

The Eisenstein elements of modular symbols for level product of two 257 distinct odd primes

DEBARGHA BANERJEE and SRILAKSHMI KRISHNAMOORTHY

Primitively generated Hall algebras

ARKADY BERENSTEIN and JACOB GREENSTEIN

Generalized splines on arbitrary graphs

Simcha Gilbert, JULiAnNA TyMOCZKO and SHIRA ViEL

Good traces for not necessarily simple dimension groups

DAVID HANDELMAN

On Fourier coefficients of certain residual representations of symplectic groups

DIHUA JIANG and BAIYING LIU

On the existence of central fans of capillary surfaces

AMMAR KHANFER

Surfaces of prescribed mean curvature $H(x, y, z)$ with one-to-one central projection onto a plane

FRIEDRICH SAUVIGNY 